\title{
Norois
}

Environnement, aménagement, société

$215 \mid 2010 / 2$

Impacts morphogéniques des tempêtes

\section{Aspects météo-marins de la tempête du 10 mars 2008 en Atlantique et en Manche}

Atmospheric and marine aspects of the 10th of March 2008 storm in Atlantic and in the Channel

Jean-Marie Cariolet, Stéphane Costa, Rémi Caspar, Fabrice Ardhuin, Rudy Magne et Gérard Goasguen

\section{(2) OpenEdition}

\section{Journals}

Édition électronique

URL : http://journals.openedition.org/norois/3242

DOI : $10.4000 /$ norois.3242

ISBN : 978-2-7535-1563-5

ISSN : $1760-8546$

Éditeur

Presses universitaires de Rennes

Édition imprimée

Date de publication : 30 septembre 2010

Pagination : 11-31

ISBN : 978-2-7535-1164-4

ISSN : 0029-182X

\section{Référence électronique}

Jean-Marie Cariolet, Stéphane Costa, Rémi Caspar, Fabrice Ardhuin, Rudy Magne et Gérard Goasguen, "Aspects météo-marins de la tempête du 10 mars 2008 en Atlantique et en Manche », Norois [En ligne], 215 | 2010/2, mis en ligne le 01 septembre 2012, consulté le 01 mai 2019. URL : http:// journals.openedition.org/norois/3242 ; DOI : 10.4000/norois.3242 


\title{
ASPECTS MÉTÉO-MARINS DE LA TEMPÊTE DU IO MARS 2008 en Atlantique et en Manche
}

\author{
Jean-Marie Cariolet \\ GÉOMER - UMR 6554 CNRS LETG \\ (Université de Bretagne Occidentale), \\ Institut Universitaire Européen de la Mer \\ Technopôle Brest-Iroise, Place Nicolas-Copernic - 29285 Plouzané \\ jean-marie.cariolet@univ-brest.fr \\ StÉPHANe Costa \\ GÉOPHEN - UMR 6554 CNRS LETG \\ (Université de Caen Basse Normandie), \\ Esplanade de la Paix, BP 8156 - 14032 CaEn Cedex, France \\ stephane.costa@unicaen.fr \\ RÉmi Caspar \\ Météo-France \\ Station météorologique du Havre, Quai des Abeilles - 76600 Le Havre, France \\ remi.caspar@meteo.fr \\ Fabrice Ardhuin, Rudy Magne \\ Service Hydrographique et Océanographique de la Marine \\ 13 rue du Chatellier, CS 92803 - 29228 Brest Cedex 2, France \\ fabrice.ardhuin@shom.fr,rudy.magne@shom.fr \\ Gérard Goasguen \\ CETMEF \\ Technopôle Brest Iroise, 155 rue Pierre-Bouguer, BP 5 - 29280 Plouzané, France \\ gerard.goasguen@developpement-durable.gouv.fr
}

\section{RÉSUMÉ}

Le 10 mars 2008, une tempête a touché la pointe nord-ouest de l'Europe pendant une marée de vive-eau, générant de nombreux dégâts et des cas de submersion sur les côtes nord-ouest de la France. Les niveaux d'eau exceptionnels atteints ce jour-là sont dus à la combinaison de deux phénomènes indépendants (surcote importante accompagnée d'une mer énorme et pleine mer de vive-eau). Les niveaux d'eau maximums à l'origine de la submersion des côtes basses ont été à chaque fois précédés par le passage d'un front froid. Les vitesses de vent et les hauteurs de houles atteintes pendant cette tempête ont une période de retour de deux ans. Les niveaux d'eau mesurés par les marégraphes ont une occurrence de deux à cent ans. Il est en revanche impossible d'estimer pour le moment la période de retour de la combinaison houle - surcote - pleine mer de vive-eau, mais elle est probablement de l'ordre de 10 ans ou plus.

MOTS CLÉ : tempête - surcote - onde de tempête - front froid - submersion 


\section{ABSTRACT \\ Atmospheric and marine aspects of the 10th of March 2008 storm in Atlan- tic and in the Channel}

The 10th of march 2008, a storm reached north-western of Europe during a spring tide, generating many damages and coastal flooding on the north-western french coast. The extrem water levels recorded on that day are caused by a combinaison of independant phenomena (important storm surge, phenomenal waves, spring high tide). The maximum water levels and most coastal flooding were every time linked with a cold front. Wind speed and wave height reached during this storm have a return period of only two years. The water levels mesured by tidal ganges have a return period from two to hundred years. Nevertheless it is impossible for the moment to estimate the return period of the combinaison wave - surge - spring high tide, but it is likely of the order of ten years or more.

KEY WORDS : storm, storm surge, cold front, inundation

Depuis quelques années, de nombreuses recherches ${ }^{1}$ ont permis de proposer un nouveau modèle explicatif du fonctionnement des perturbations atlantiques (Joly, 1995). D'après ce modèle, qui prend en considération toute la troposphère, les dépressions se déplacent d'ouest vers l'est le long d'un rail appelé « rail des dépressions ». L'étude des trajectoires des dépressions Atlantiques montre qu'il existe deux types de configurations. Dans la première, le rail qui part toujours de la région de Terre-Neuve, se coupe en deux au niveau du $30^{\mathrm{e}}$ méridien ouest pour se diriger vers l'Islande ou vers l'Espagne. Dans la seconde, le rail s'étend le long du 50e parallèle jusqu'aux îles Britanniques (fig. 1). Ce dernier cas de figure est le plus fréquent (Bessemoulin, 2002; Beltrando, 2004).

Ces recherches ont également permis d'identifier le rôle des courants-jets, courants d'altitude générés par la différence de température entre les pôles et l'équateur. Les courants-jets circulent d'ouest en est au niveau des régions tempérées (latitude $\left.50-55^{\circ} \mathrm{N}\right)$, dans la haute troposphère $(8-10 \mathrm{~km}$ d'altitude), et favorisent la formation et le creusement des dépressions. Ce phénomène s'explique en partie par le mécanisme d'interaction barocline, défini comme étant l'amplification de deux anomalies (une anomalie froide située en altitude et une anomalie de surface à savoir une dépression) qui interfèrent avec le courant jet (Ayrault et Joly, 2000). La région la plus favorable à l'amplification des tempêtes semblerait se situer à l'extrémité est du jet (fig. 1B). Autrement dit, en configuration zonale, les dépressions coupent souvent le courant jet au niveau du $15^{\mathrm{e}}$ méridien ouest, et s'en trouvent alors amplifiées par le mécanisme d'interaction barocline (Ayrault et Joly, 2000; Bessemoulin, 2002).

L'arrivée d'un système dépressionnaire par l'Atlantique sur les côtes du Nord-Ouest de la France génère très souvent un phénomène de surcote, défini comme étant une surélévation temporaire du niveau de la mer. Ce phénomène peut être dû à une baisse des pressions atmosphériques qui produit une élévation du niveau d'eau, et/ou à des vents que l'on peut qualifier d'afflux, car ils soufflent de la mer vers la côte et favorisent ainsi l'amoncellement de l'eau sur les côtes (Bouligand et Tabeaud, 1998, Pirazzoli et al., 2006; Caspar et al., 2007). Les météorologues parlent de marée de tempête ou onde de tempête pour désigner le niveau d'eau exceptionnel résultant de la conjonction d'une surcote avec la marée astronomique. Les dépressions d'origine Atlantique s'accompagnent également très souvent d'une forte agitation marine - agitation due à l'arrivée d'une houle générée au large, ou due à l'effet local du vent sur la mer voire à une combinaison des deux - ce qui contribue à la surélévation du niveau d'eau moyen à la côte (surcote induite par les vagues ou «wave setup ", voir par exemple M. S. Longuet-Higgins et R. W. Stewart, 1963). Par ailleurs, les vagues de grandes périodes générées par fortes tempêtes, localement ou à distance, sont aussi

1. Nous pouvons citer notamment le programme FASTEX (Fronts and Atlantic Storm-Track Experiment), expérience sur le rail des dépressions atlantique et les fronts. 

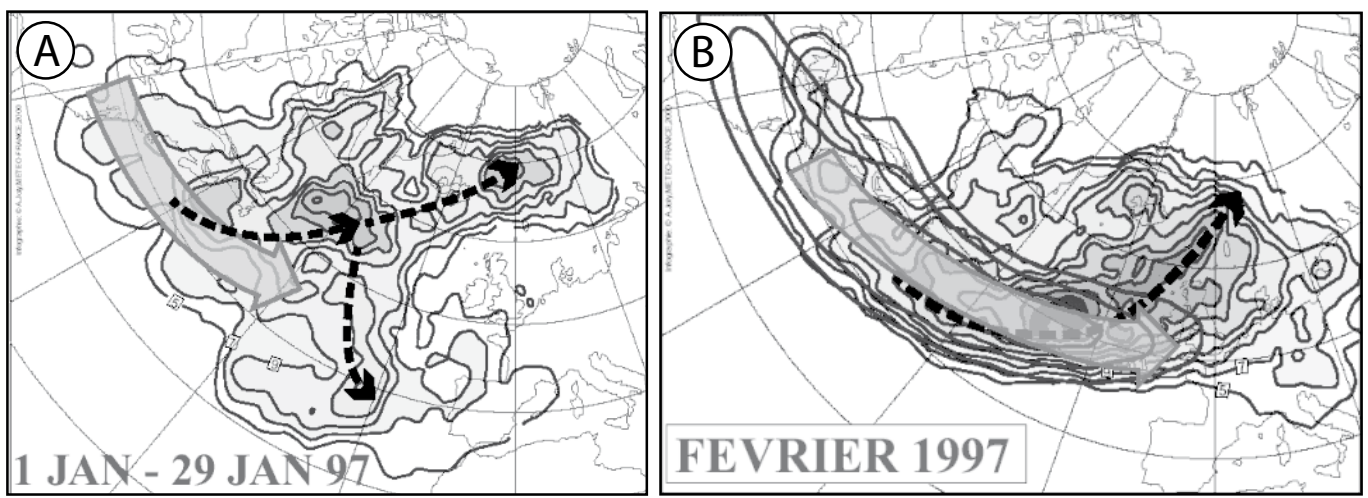

- Trajectoire type suivie par les dépressions

Symbolisation du flux moyen en altitude (courant jet)

Figure 1:A : Le rail des dépressions en janvier 1997. Nous sommes ici en configuration de blocage : les tempêtes restent sur l'Atlantique. Le courant-jet, courant d'altitude, est limité à la partie ouest de l'océan Atlantique. D'après C. Baehr et al., 1999 (source : http://www.cnrm.meteo.fr/dbfastex/recyf_temp/tempet003.html\#railbloc). B : Le rail des dépressions en février 1997. Dans cette configuration dite «zonale », le rail des dépressions s'étend le long du $50^{\mathrm{e}}$ parallèle. Les dépressions ne sont pas bloquées. On remarque que le courant-jet s'étend plus à l'est et que les dépressions ont tendance à couper le courant-jet du côté de son extrémité est. D'après C. Baehr et al., 1999. (source : http://www.cnrm.meteo.fr/dbfastex/recyf_temp/tempet004.html\#railzonal)

A: Storm track in January 1997. We are here in a blocking pattern: storms stay on the Atlantic. The jet stream is limited at the western part of the Atlantic ocean. After Baehr et al., 1999. B: Storm track in february 1997. In this zonal pattern, storm tracks go along the 50th parallel. Depressions are not blocked. We can observe that the jet stream go far east and that depressions tend to cut the jet stream around its eastern extremity. After Baehr et al., 1999.

la source d'ondes longues de quelques minutes de période, qui sont la cause des mouvements de seiche dans la plupart des ports (Okihiro et al., 1993) et peuvent engendrer des modulations du niveau d'eau d'amplitude métrique, à l'échelle de quelques minutes. Ces oscillations peuvent ainsi contribuer de façon significative aux dégâts côtiers.

En milieu macrotidal, ces différents phénomènes, qui contribuent à l'élévation temporaire du niveau d'eau à la côte, ne présentent un risque pour la zone littorale que lorsqu'ils se produisent pendant une pleine mer de vive-eau. Sur nos côtes, le caractère exceptionnel et destructeur d'un tel événement résulte donc de la combinaison rare de deux phénomènes indépendants : une marée de vive-eau, phénomène astronomique, et une onde de tempête, phénomène météorologique. En 2000, P. A. Pirazzoli indiquait que, selon les probabilités statistiques, la menace qu'un événement tempétueux se combine à une marée de vive-eau sur la côte nord Atlantique était de plus en plus probable. Cette hypothèse s'est vérifiée le 10 mars 2008, lorsqu'une onde de tempête - accompagnée d'une houle d'origine Atlantique - a touché la pointe nord-ouest de la France alors que les coefficients de marée étaient de 106 et 104.

La tempête qui a touché la pointe ouest européenne ce jour-là, nommée " Johanna », a eu un impact très important sur l'ensemble du littoral du Nord-Ouest de la France (breton et normand) : plus d'une trentaine d'inondations par la mer recensés de Sarzeau (Morbihan) à Cayeux sur mer (Somme), des reculs du trait de côte impressionnants dans le Finistère et de nombreux dommages sur les ouvrages de défense, sur les infrastructures portuaires et sur les habitations ont été observés ${ }^{2}$. Cet épisode peut être qualifié d'exceptionnel de par l'étendue du linéaire côtier

2. Source : demandes de reconnaissance de catastrophe naturelle faites par les communes après le passage de la tempête, et les quotidiens locaux Ouest-France, Le Télégramme, Le Trégor. 
touché et également du fait du nombre important de sites atteints, comme cela sera abordé plus loin. Si les tempêtes historiques de l'hiver 1989-1990 avaient été plus violentes, elles n'avaient en effet affecté respectivement que le département du Finistère et le littoral de la Manche (Fichaut et Hallégouët, 1989; Caspar, 1990; Hallégouët et Bodéré, 1993; Regrain, 1992; Costa, 1995 ; Héquette et Vasseur, 1998; Tonnerre, 2001 ; Daniel, 2001 ; Hallégouët et Hénaff, 2006 ; Caspar et al., 2007). D’un point de vue purement anémométrique, la tempête « Johanna » ne fait pas partie des tempêtes les plus violentes qu'a connu la façade atlantique française ces dernières décennies. Les plus fortes rafales enregistrées lors du passage de la tempête ont atteint $150 \mathrm{~km} / \mathrm{h}$ sur la pointe finistérienne (Léry, 2008). De telles vitesses de vents se produisent en moyenne une fois tous les deux ou trois ans dans le Nord-Ouest de la France (Léry, 2008). Des valeurs bien supérieures de 180 à $200 \mathrm{~km} / \mathrm{h}$ ont été enregistrées lors des tempêtes de décembre 1999, février 1990 et octobre 1987 (Santurette, 2001).

S. Costa et al. (2004), R. Caspar et al. (2007) ont montré que sur les côtes de la Manche, il existe un lien entre le passage d'un front froid et la submersion marine. Un front peut être défini comme étant une "zone de discontinuité de température et de vent en surface, qui s'accompagne de précipitations et de vents forts » (Santurette, 2001). Un front froid, zone météorologiquement active, désigne la limite entre une masse d'air froid située en arrière, et une masse d'air chaud soulevée par la première (Beltrando, 2004). Nous verrons en quoi le front froid a joué un rôle au cours de la tempête du 10 mars 2008. Au-delà des conditions atmosphériques et hydrodynamiques, le budget sédimentaire des plages, et à travers lui la morphologie des cordons littoraux, contrôle également l'ampleur des dégâts (Orford, 1977; Paskoff, 1985; Carter, 1988; Carter et Orford, 1993 ; Costa, 1997 ; Morton R. A., 2002 ; Sabatier et al., 2009). Des conditions d'agitation même peu énergétiques mais fréquentes (notamment durant la période hivernale) commandent la morphologie «pré-tempête » de la plage marquée par un abaissement de son profil, ce qui peut alors renforcer l'impact morphogénique d'une tempête.

Afin de mieux comprendre et d'expliquer l'ampleur du phénomène qui a touché les littoraux du Nord-Ouest de la France le 10 mars 2008, cet article propose de revenir tout d'abord sur les conditions météo-atmosphériques observées lors de cet événement, puis d'analyser les données météo-marines enregistrées sur les littoraux du Nord-Ouest de la France (fig. 2). Une dernière partie fera un bref état des lieux des différents impacts morphogéniques qui ont été générés par le passage de la tempête. Quelques sites mentionnés dans cette dernière partie feront l'objet d'études de cas plus approfondies qui seront traités dans ce numéro.

\section{Dynamiques météo-atmosphériques}

\section{SitUATION EN SURFACE}

Le soir du samedi 8 mars 2008, quelques jours avant que la tempête ne touche les côtes de l'ouest de la France, une dépression prend naissance au niveau de Terre-Neuve, avec une pression en son centre de $1010 \mathrm{hPa}$. Elle se déplace assez lentement le long du $55^{\mathrm{e}}$ parallèle nord et atteint l'Irlande le 10 mars à 06 h 00 UTC (fig. 3 et 4A). La trajectoire de la dépression est conforme à la configuration la plus fréquente du rail des dépressions, dite «zonale» (fig. 1 et 3 ). La tempête s'est creusée à un rythme régulier d'environ 10 à 15 hPa toutes les 24 heures, ce qui ne représente pas un creusement rapide. Pour exemple, lors de la tempête du 24-25 janvier 1990, une baisse de 36 hPa en 24 heures avait été observée (Tonnerre-Guérin, 2003).

Le matin du 10 mars, la pression au centre de la dépression alors située sur l'Irlande, est de $953 \mathrm{hPa}$. Au même moment, la pression enregistrée au sud de la Bretagne est de $980 \mathrm{hPa}$. Le gradient de pression sud-ouest est alors de $30 \mathrm{hPa}$ sur seulement 600 kilomètres de distance (fig. 4A).

En analysant les données de vent et de pression atmosphérique au niveau de l'île d'Ouessant (fig. 5), on remarque le passage d'un front froid le matin du 10 mars. Avant le passage de ce dernier, la pression atmosphérique diminue rapidement jusqu’à $975 \mathrm{hPa}$ et le vent de secteur sud-ouest 


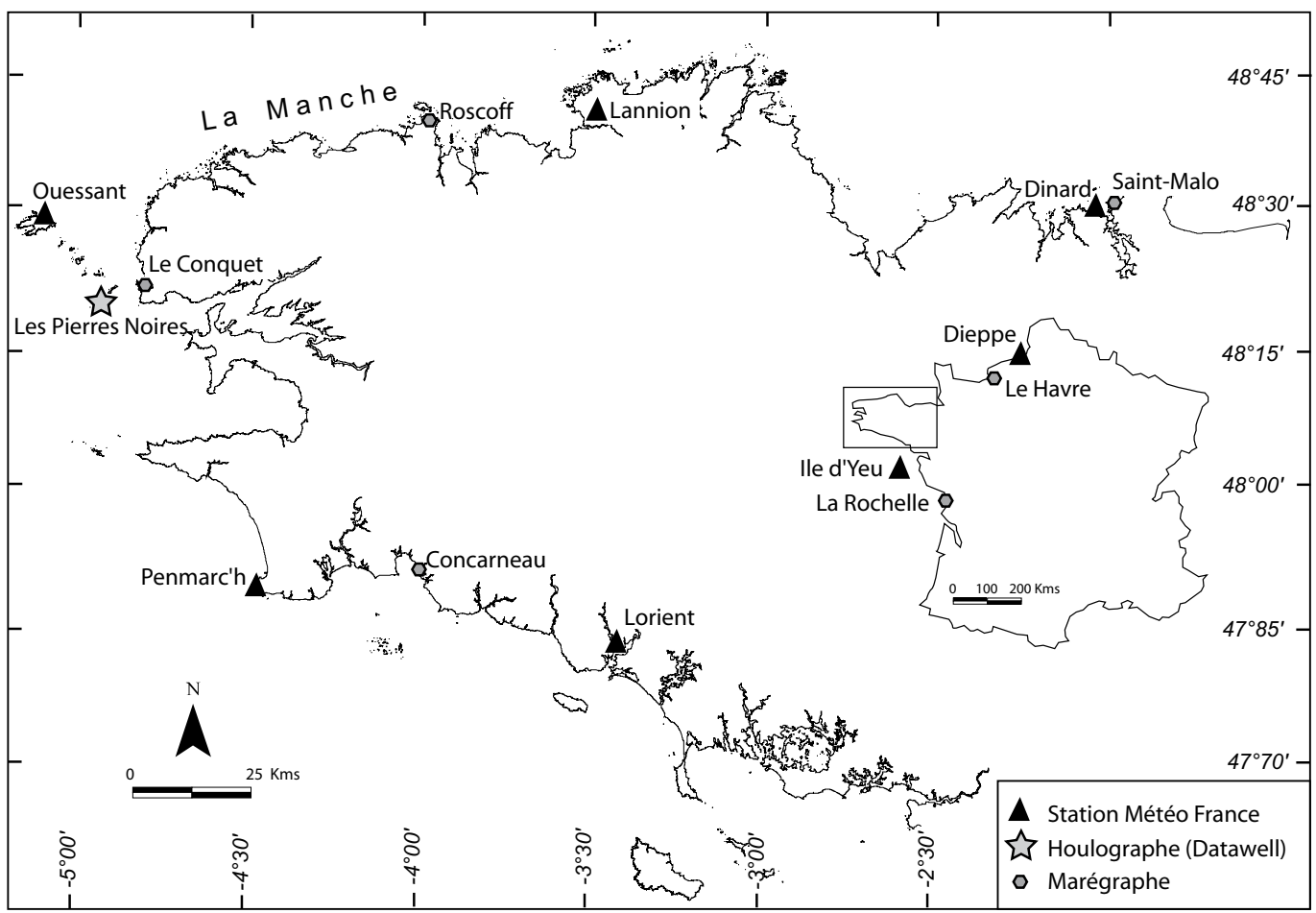

Figure 2 : Carte de localisation des stations Météo France, des marégraphes et du houlographe dont les données ont été utilisées lors de cette étude used in this paper

Location map of Meteo France weather stations, tidal ganges and wave gauge that data has been

Figure 3 : Trajectoire de la dépression «Johanna » et situation synoptique au 10 mars 2008 à 06 h 00 UTC La dépression n'a pas été bloquée car aucun anticyclone n'était présent sur l'Europe. La configuration, avec un anticyclone situé au milieu de l'Atlantique vers le $35^{\circ} \mathrm{N}$ et une dépression vers le $55^{\circ} \mathrm{N}$, est zonale (source : Météo-France).

Storm-track of

"Johanna" and synoptic situation the 10th of march at 06:00 UTC

The depression is not blocked because no anticyclon is based on Europe. The pattern, with an anticyclon located on Atlantic around the $35^{\circ} \mathrm{N}$ and $a$ depression around the $55^{\circ} \mathrm{N}$, is zonal (source : Météo-France).

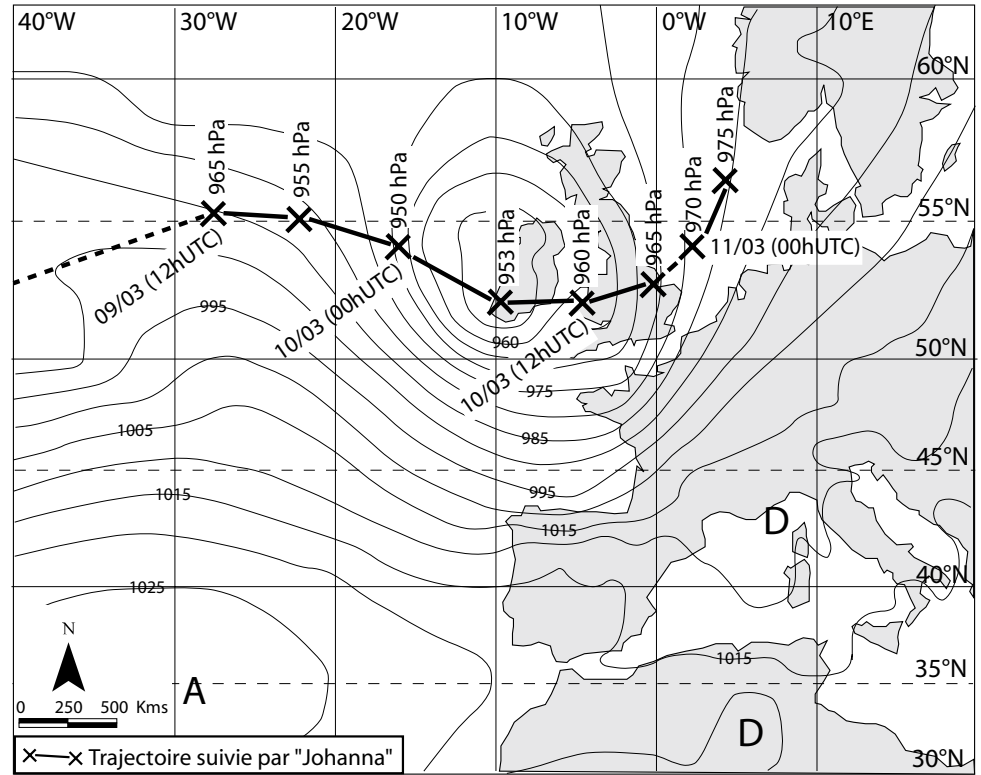



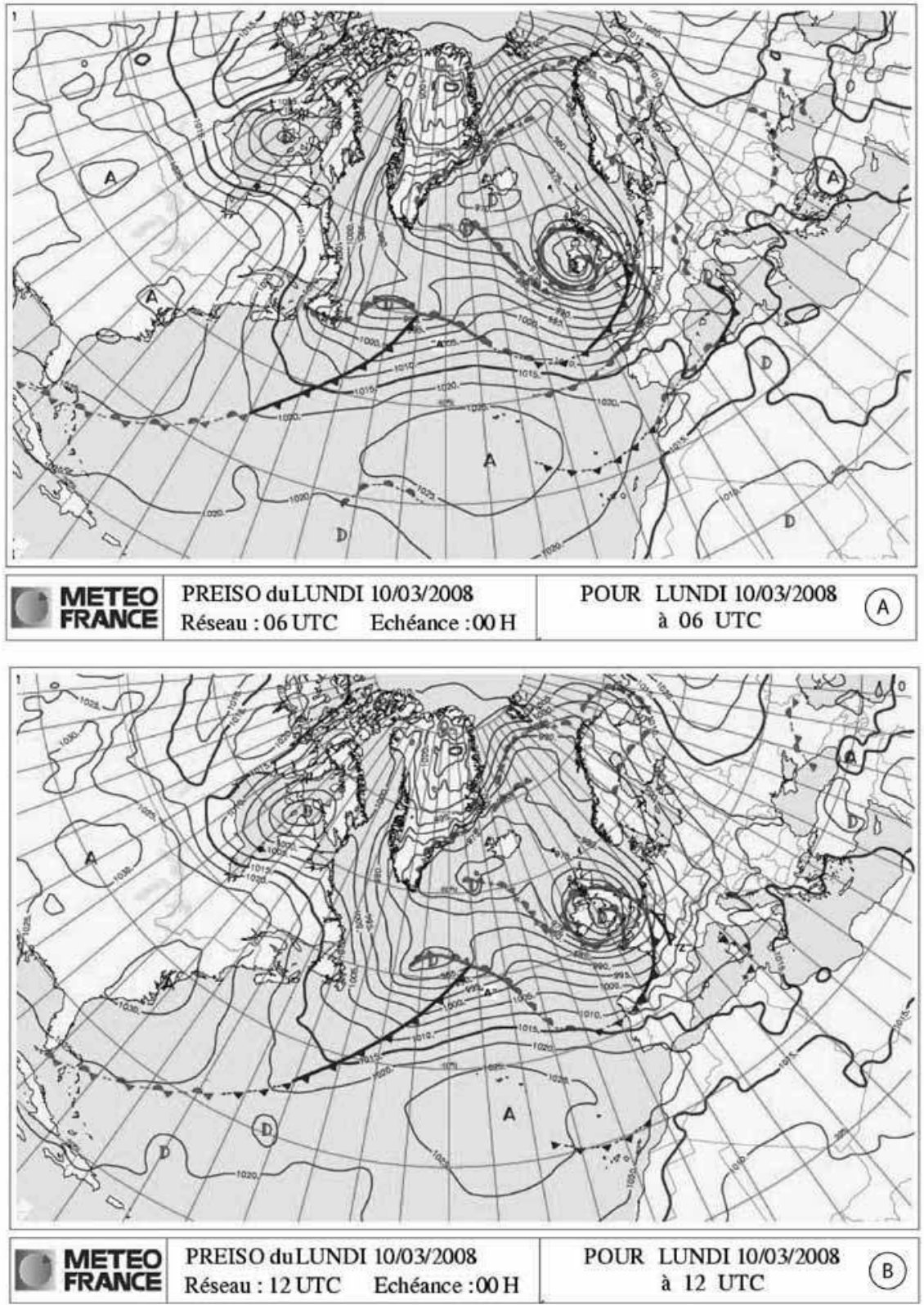

Figure 4 : Évolution synoptique entre 12 h 00 UTC le 10 mars et 00 h00 UTC le 11 mars 2008 (source : MétéoFrance)

A : 06 h00 UTC : une heure après la pleine mer et les submersions sur la côte sud de la Bretagne, la dépression atteint l'Irlande avec une pression en son centre de $953 \mathrm{hPa}$. Le front froid « principal » vient de traverser la Bretagne. B : 12 h 00 UTC : la dépression progresse lentement sur la mer d'Irlande. La perturbation principale pénètre en Belgique. Un segment d'occlusion traverse la Manche occidentale. C : 18 h00 UTC : une heure avant la pleine mer et les submersions sur la côte nord de la Bretagne. La dépression, encore vigoureuse (965 hPa), se situe un peu au nord du bassin de Londres. Un petit front froid «secondaire » est identifié sur les côtes de la Seine-Maritime. $\mathrm{D}$ : 00 h 00 UTC le 11 mars : à l'heure approximative de la pleine mer de Dieppe et donc des submersions de la côte d'Albâtre, la dépression est en mer du Nord, et le petit front froid «secondaire » traverse le Benelux. En Manche orientale, les isobares s'alignent peu à peu sur un axe nord-ouest/sud-est. 

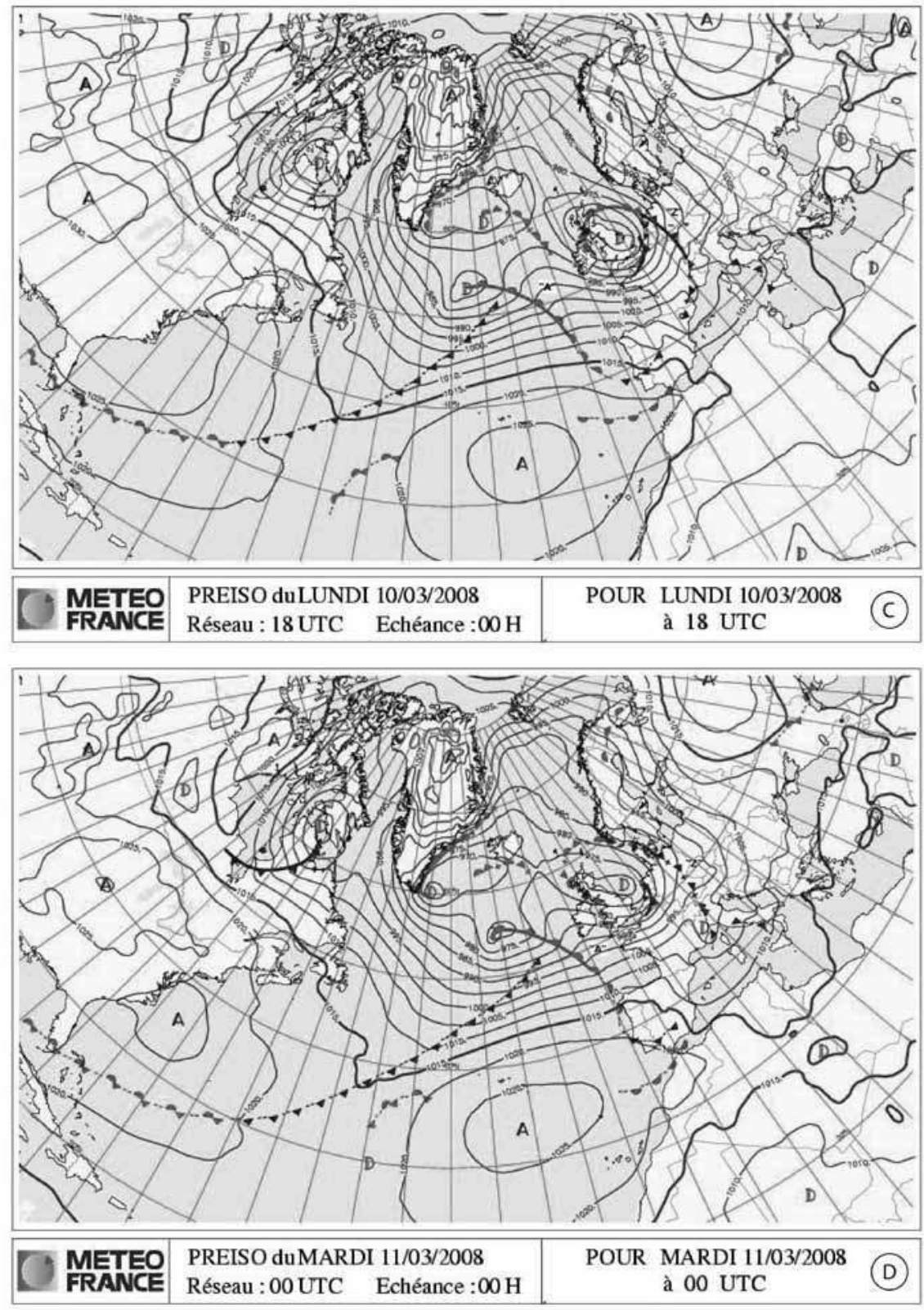

Figure 4: Synoptic evolution between 12:00 UTC the 10th of march and 00:00 UTC the 11 of march 2008 (source : Météo-France)

A : 06:00 UTC : an hour after high tide and floodings on south coast of Brittany, the depression reach Irland with a central pressure of $953 \mathrm{hPa}$. The "main" Cold front has just crossed Brittany. B : 12:00 UTC : the depression move slowly on Irish sea. C : 18:00 UTC : an hour before high tide and floodings on north coast of Brittany. The depression (965 $\mathrm{hPa}$ ), is located at the north of London basin. A minor cold front is indentified on the coasts of Seine-Maritime. D : 00:00 UTC le 11 mars : during high tide at Dieppe and floodings on the coast of Albatre, the depression is on the North Sea and the minor cold front cross Benelux. In the eastern Channel, isobars align gradualy with a north-west / south-est axis. 

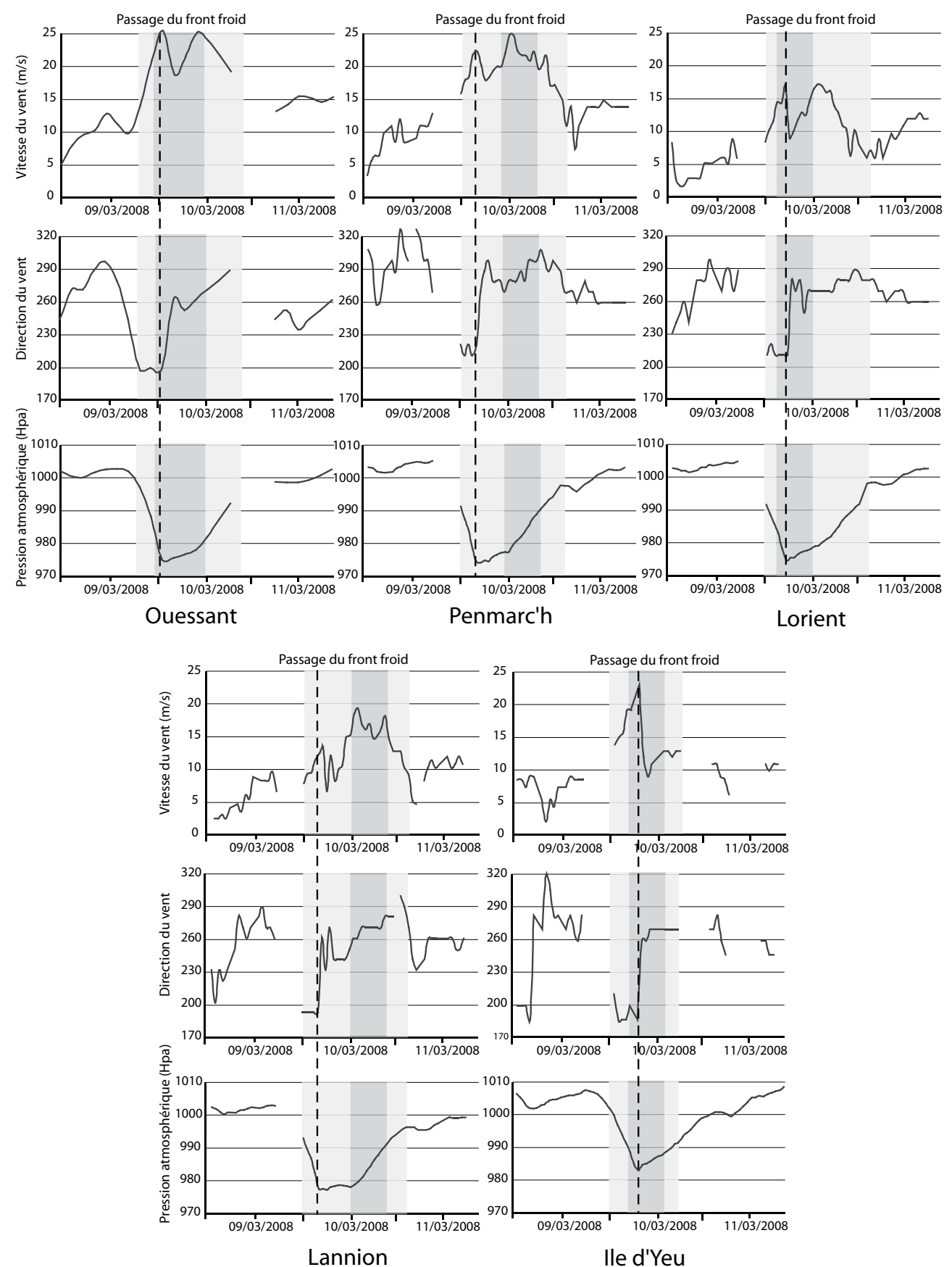

Figure 5 : Conditions de vent et de pression atmosphérique enregistrées dans les stations de Penmarc'h, Lorient, Lannion et de l'île d'Yeu entre le 9 mars et le 11 mars 2008

On remarque le passage du front froid le matin du 10 mars pour les quatre stations (source : Météo-France).

Wind and atmospheric pressure conditions recorded at the weather stations of Penmarc'h, Lorient,

Lannion and île d'Yeu between the $9^{\text {th }}$ and the $11^{\text {th }}$ of march 2008

We can see the passing of a cold front the $10^{\text {th }}$ of march in the morning for the 4 stations (source : Météo-

France). 
forcit. Suite au passage du front froid, la vitesse du vent chute brusquement. La pression atmosphérique remonte ensuite progressivement et le vent passe au secteur ouest puis nord-ouest en forcissant à nouveau. On retrouve le même schéma pour les stations météorologiques de Penmarc'h, Lannion et Lorient où le front froid est passé respectivement à 4h 00, 4 h 30 et 5 h 00 UTC (fig. 5).

Au soir du 10 mars, le centre dépressionnaire s'est déplacé au sud-est de l'Angleterre et la dépression n'a pas diminué en intensité (fig. 4C). À 19 heures UTC, la pression au centre du système dépressionnaire est de $960 \mathrm{hPa}$ et celle mesurée au nord de la Bretagne est de $985 \mathrm{hPa}$ (fig. 4C). Le gradient de pression nord-ouest est alors de $25 \mathrm{hPa}$ sur une distance de $500 \mathrm{~km}$. Cette situation se traduit par des vents toujours violents mais orientés maintenant au secteur ouest-nord-ouest.

Après le passage du front froid « secondaire » sur les côtes de la Seine-Maritime, vers 18 heures UTC, on ne détecte plus d'activité frontologique en Manche orientale durant la nuit, ni sur les documents de Météo-France, ni à l'examen des «données minute » du sémaphore de Dieppe (fig. 6). Il n'existe alors plus de forme météorologique active (front froid actif ou dépression creuse en mouvement rapide) même si la dépression en cause reste vigoureuse sur le sud des îles Britanniques. Le gradient de pression barométrique sur la Manche est assez remarquable à $00 \mathrm{~h}$ UTC le 11 mars (horaire proche de la pleine mer théorique sur la côte d'Albâtre, au moment des submersions) : de niveau 5 dans l'échelle des « indices de gradient de nord-ouest » définie par S. Costa (1997), soit 5 intervalles de $5 \mathrm{hPa}$ le long d'un transect s'étirant de Cromer (sud-est de l'Angleterre) à la Pointe du Raz. L'indice 6 étant le degré maximal observé lors des submersions exceptionnelles de février 1990.

Direction du vent, pression station et température, à Dieppe, du 10 mars 2008, 16 h UTC, au 11 mars 2008, 01 h UTC

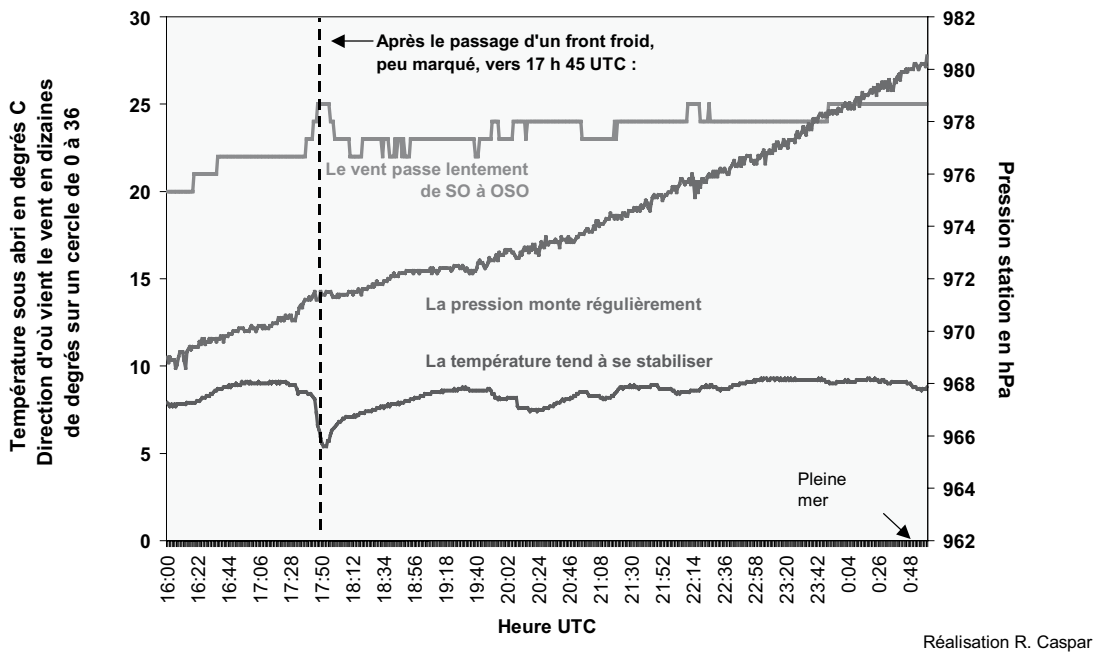

Figure 6 : Évolution des paramètres météorologiques sur le site de Dieppe après le passage d'un petit front froid secondaire le 10 mars 2008 vers $17 \mathrm{~h} 45$ UTC

Sur l'échelle de gauche, on lit à la fois les valeurs de la température sous abri, en degrés C, et celles de la direction du vent, en dizaines de degrés sur un cercle de 0 à 36. La pleine mer en cause dans les submersions est indiquée sur le graphe (Source : Météo-France [Caspar, 2009]).

Meteorological parameters evolution for the site of Dieppe after a little cold front passing the 10th of march 2008 at around 17:45 UTC

On the left scale, we read temperature $\left({ }^{\circ} \mathrm{C}\right)$ under shelter, and wind direction (ten degrees on a circle from 0 to 36). The high tide which made inundations is indicated on the graph. (Source : Météo-France [Caspar, 2009]). 
Jean-Marie Cariolet et AL.

Durant le flot de la nuit du 10 au 11 mars, le vent passe progressivement de sud-ouest à ouest sur les côtes de la Haute-Normandie et celles de Picardie (il prend une composante dite « d'afflux »). Des interactions complexes se mettent en place, entre l'onde de marée, la puissance des vagues qui augmente, les surcotes d'ordre météorologique et de déferlement... qui aboutissent localement à des submersions sévères.

Au cours de la journée du 11 mars, la dépression se comble progressivement en atteignant la mer du Nord, vers le $55^{\mathrm{e}}$ parallèle nord (fig. 3). La tempête est terminée, mais les dégâts le long des côtes bretonnes et de la Manche sont importants. Selon Météo-France, les vitesses de vents atteintes pendant la tempête ont une occurrence de deux à trois ans. Cependant, la durée de l'épisode tempétueux est inhabituelle. Les vents violents ont en effet soufflé pendant une douzaine d’heures (Léry, 2008).

\section{SituATiOn EN ALTITUdE}

Au regard de la situation en altitude, plus précisément au niveau de la limite entre la troposphère et la stratosphère, on remarque un tourbillon situé à quelques centaines de km à l'ouest de la dépression située en surface, le 9 mars 2008 à 6 heures UTC (fig. 7A - planche I). Cette anomalie d'altitude a pu alimenter la dépression (anomalie de surface) lorsque cette dernière a coupé le courant-jet, zone de forts vents située à 200 hPa (fig. 7A - planche I). Autrement dit, les deux anomalies se sont vraisemblablement couplées avec le courant-jet, et se sont amplifiées de façon mutuelle par le mécanisme d'interaction barocline (fig. 8). C'est par ce mécanisme que la dépression a pu se creuser pour devenir tempête (Ayrault et Joly, 2000). Si l'on observe maintenant la situation de la surface isobarique à 850 hPa (située à environ 1500 m d'altitude) du 9 mars 2008 (fig. 7B - planche I), on remarque que les isothermes (lignes d'égale température) sont fortement resserrées au niveau de la côte est du Canada (région de Terre Neuve). L'isotherme $-30{ }^{\circ} \mathrm{C}$ se situe à seulement 1100 kilomètres de l'isotherme $16{ }^{\circ} \mathrm{C}$. Une différence de température de $46{ }^{\circ} \mathrm{C}$ sur une distance aussi "faible » a vraisemblablement créé un puissant courant-jet qui a favorisé le creusement de la dépression (fig. $7 \mathrm{~B}$ - planche $\mathbf{I}$ ).

À une échelle plus locale, l'analyse de la situation en altitude permet d'expliquer la présence d'une « traîne » active (fig. 9 - planche II) qui s'est déployée sur les côtes de la Manche le 10 mars, quelques heures après le passage du front froid « secondaire » visible sur la figure $4 \mathrm{C}$. Celle-ci est due à un petit «forçage » d'altitude (fig. 10 - planche II) s'exprimant par l'advection - du Pays de Galles à la baie de Somme, entre 21 heures et 24 heures UTC - d'une « bulle » relativement froide vers $500 \mathrm{hPa}$, d'environ -30 degrés (l'environnement à ce niveau étant déjà très froid). Cette survenue d'air relativement plus froid en altitude, au sein d'une masse d'air déjà instable, a pour conséquence un accroissement des rafales de vent en force et fréquence alors que le flot commence, ce qui habituellement génère d'un point de vue océanographique une augmentation de l'amplitude des vagues de « la mer du vent ».

\section{Conditions météomarines}

\section{UNE SURCOTE IMPORTANTE}

Deux cycles de marées se sont succédés pendant le passage de la tempête, avec une pleine mer théorique le matin et une le soir sur les côtes bretonnes (le midi du 10 mars et dans la nuit du 10 au 11 mars pour les régions Haute-Normandie et Picardie). Les coefficients de marée étaient alors de 106 (le matin) et 104 (le soir) (tableau 1). 


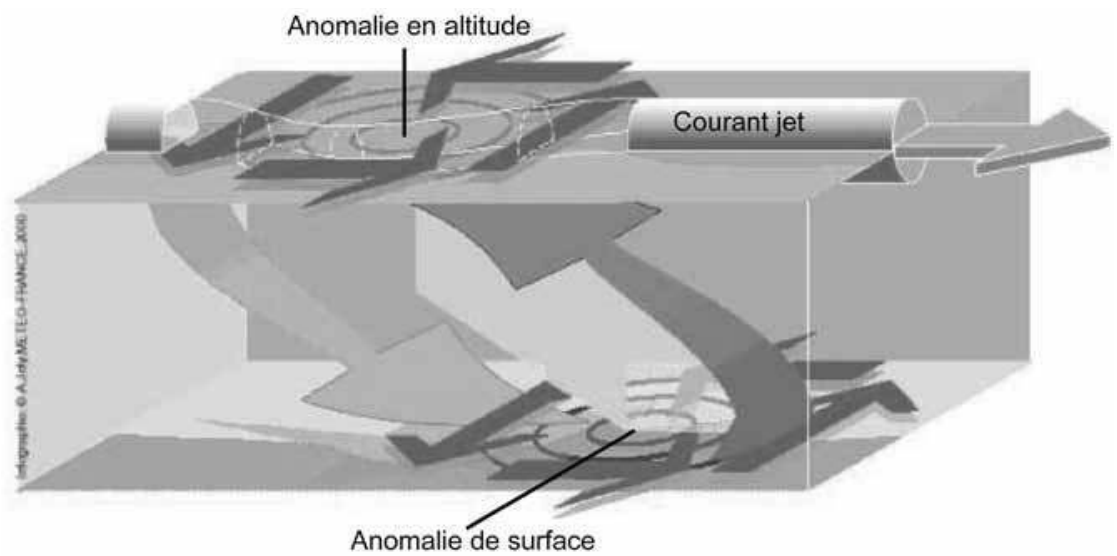

Figure 8 : Représentation d'un courant-jet rectiligne simplifié

Ce schéma illustre bien le principe d'interaction barocline : une anomalie froide située en altitude alimente une anomalie de surface (dépression) située plus à l'est. Les deux anomalies sont couplées avec le courant-jet et s'amplifient de façon mutuelle (Source : http://france.meteofrance.com/content/2008/4/3313-43.jpg).

Diagram of a simplified and straight jet stream

This diagram show well the principle of baroclinic interaction : a cold anomaly situated in altitude supply an anomaly situated on the surface (depression). Both anomalies are coupled with the jet stream and intensify each other.

\begin{tabular}{|c|c|c|c|c|c|c|}
\hline \multirow[b]{2}{*}{ Marégraphes } & \multicolumn{4}{|c|}{10 mars 2008} & \multicolumn{2}{|c|}{11 mars 2008} \\
\hline & matin & $\begin{array}{l}\text { hauteur } \\
\text { (SHOM) }\end{array}$ & soir & $\begin{array}{l}\text { hauteur } \\
\text { (SHOM) }\end{array}$ & matin & $\begin{array}{l}\text { hauteur } \\
\text { (SHOM) }\end{array}$ \\
\hline Le Conquet & $5 \mathrm{~h} 37$ & $7,26 \mathrm{~m}$ & 17 h 52 & $7 \mathrm{~m}$ & $6 \mathrm{~h} 15$ & $7,08 \mathrm{~m}$ \\
\hline Concarneau & $5 \mathrm{~h} 08$ & $5,34 \mathrm{~m}$ & $17 \mathrm{~h} 21$ & $5,1 \mathrm{~m}$ & $5 \mathrm{~h} 47$ & $5,17 \mathrm{~m}$ \\
\hline Roscoff & $6 \mathrm{~h} 47$ & $9,35 \mathrm{~m}$ & $19 \mathrm{~h} 05$ & $9,07 \mathrm{~m}$ & $7 \mathrm{~h} 24$ & $9,15 \mathrm{~m}$ \\
\hline Saint-Malo & $7 \mathrm{~h} 57$ & $12,81 \mathrm{~m}$ & $20 \mathrm{~h} 14$ & $12,47 \mathrm{~m}$ & $8 \mathrm{~h} 33$ & $12,54 \mathrm{~m}$ \\
\hline Sables-d'Olonne & $5 \mathrm{~h} 09$ & $5,5 \mathrm{~m}$ & $17 \mathrm{~h} 25$ & $5,24 \mathrm{~m}$ & $5 \mathrm{~h} 48$ & $5,31 \mathrm{~m}$ \\
\hline Le Havre & & & $12 \mathrm{~h} 03$ & $8,13 \mathrm{~m}$ & oh 29 & $7,95 \mathrm{~m}$ \\
\hline
\end{tabular}

Tableau 1 : Horaires (UTC) et hauteurs d'eau prédites (altitudes SHOM) aux pleines mers du 10 mars et du 11 mars 2008 pour les marégraphes du Conquet, de Concarneau, Roscoff, Saint-Malo, des Sables-d'Olonne et du Havre (source : $\mathrm{SHOM}$ )

Schedule (UTC) and predicted water heights (SHOM) at the high tides of the $10^{\text {th }}$ and the $11^{\text {th }}$ of march 2008 for the tidal gange of Le Conquet, Concarneau, Roscoff, Saint-Malo, Les Sables-d'Olonne and Le Havre (source : $\mathrm{SHOM}$ )

Le passage de la tempête a été accompagné d'une surcote importante dont témoignent les enregistrements des marégraphes du Nord-Ouest de la France (fig. 11). La surcote maximale enregistrée lors du cycle tidal durant cet épisode a eu lieu à Saint-Malo à 16 h00 UTC, et est égale à 1,41 m. Par comparaison l'ouragan d'octobre 1987 avait généré à Brest une surcote " record » de 1,45 m (Bouligand et Tabeaud, 1998). On peut donc qualifier celle enregistrée à Saint-Malo le 10 mars 2008 d'importante. Heureusement cette valeur a été atteinte au moment de la basse mer. Quelques heures plus tard, et les dégâts sur la côte nord du département de l'Ille-et-Vilaine auraient été beaucoup plus importants. Concernant les marégraphes du Conquet, de Roscoff, de Concarneau, des Sables-d'Olonne et du Havre, les valeurs maximales atteintes lors du cycle tidal 
Jean-Marie Cariolet et AL.

ont été respectivement égales à $0,8 \mathrm{~m}$ (14h00 UTC), 0,71 m (04h00 UTC), 0,78 m (05 h 00 UTC), 0,89 m (08h00 UTC) et 1,18 m (20 h00 UTC).

En Bretagne et en Vendée, c'est durant la pleine mer du matin que la surcote a joué un rôle important. L'exemple de Concarneau l'illustre bien, la surcote maximum de 0,78 m ayant été atteinte à 05 heures UTC, heure de la pleine mer. Les vents - que l'on peut qualifier ici de vents d'afflux - soufflaient alors de sud-ouest à ouest et la pression atmosphérique était de $976 \mathrm{hPa}$ (fig. 5 - station de Penmarc'h). Les autres marégraphes bretons ont enregistré une surcote plus faible pendant la marée haute du matin, avec $0,53 \mathrm{~m}$ au Conquet, $0,57 \mathrm{~m}$ à Roscoff et $0,60 \mathrm{~m}$ à Saint-Malo. Au Sables-d'Olonne, la surcote mesurée au moment de la pleine du matin était de $0,73 \mathrm{~m}$. Elle s'est accrue avec le jusant.

Durant la pleine mer du soir, les valeurs de surcote atteintes en Bretagne ont été plus faibles, s'étalant selon un gradient sud-nord de $0,12 \mathrm{~m}$ à Concarneau à 0,43 m à Saint-Malo (fig. 11). Ceci s'explique par une remontée des pressions atmosphériques autour de $985 \mathrm{hPa}$ et par une diminution de la force du vent qui a tourné nord-ouest (fig. 5).

En Haute-Normandie, le pic de surcote a été atteint pendant la marée basse. Les valeurs de surcote pendant les pleines mers étaient en effet moins importantes : au Havre, les surcotes au moment des pleines mers du 10 mars à 12 heures UTC et du 11 mars à 01 h UTC étaient ainsi respectivement de $0,62 \mathrm{~m}$ et $0,5 \mathrm{~m}$ (fig. 11 ).

Il est important de signaler que les valeurs de surcote enregistrées dans les ports de référence ne traduisent pas la situation générale. En Bretagne notamment, où la côte est très découpée, la déformation du plan d'eau à la côte a pu être différente à certains endroits pourtant très proches. Par exemple, sur la côte occidentale du Trégor, particulièrement touchée durant la pleine mer du 10 mars au soir, l'effet des vents d'ouest qui soufflaient perpendiculairement à la côte, a sans aucun doute été plus important qu'à Roscoff (orienté vers le nord) où est localisé le marégraphe le plus proche.

Si l'on s'en tient aux cartes de niveaux d'eau extrêmes établies par le $\mathrm{SHOM}^{3}$ selon la méthode de B. Simon (1996), les niveaux d'eau maximum atteints le 10 mars 2008 ont une occurrence centennale pour le marégraphe de Concarneau (avec un niveau maximal de 3,58 m NGF), décennale pour Roscoff (avec 5,13 m NGF) où inférieure à 10 ans (Le Conquet et Saint-Malo). Concernant les valeurs de surcote, bien que qualifiées d'importantes, elles ne présentent pas de caractère exceptionnel. Ainsi, L'ouragan qui a balayé le Nord-Ouest de la France le 15 octobre 1987 avait généré pendant plusieurs heures des surcotes bien supérieures. Celles-ci avaient atteint 1,45 m et $1,70 \mathrm{~m}$ respectivement à Brest et au Havre (Hontarrède et Galli, 2001). Les coefficients de marée étaient alors faibles. De même, sur les côtes normandes, on se souvient de la journée du 22 janvier 1988 où une surcote record de 1,90 m a été enregistrée au port du Havre. Le coefficient de marée était alors de 104. Le pic de surcote fut atteint avant la pleine mer, ce qui minimisa les dégâts (Caspar, 1988).

Au final, si l'on observe les courbes marégraphiques du 10 et 11 mars 2008, on remarque que les niveaux d'eau les plus importants ont été atteints pendant la pleine mer du matin le 10 mars 2008, juste après le passage du front froid. Cependant, si de nombreux sites ont été affectés le matin, d'autres l'ont été durant la pleine mer du soir. Un autre paramètre est donc à prendre en considération, et permet d'expliquer l'ampleur des dégâts recensés le soir du 10 mars 2008 : l'arrivée d'une forte houle de l'Atlantique.

\section{Mer énorme en AtLantique et importance de la PÉriode}

Les seules mesures de l'état de la mer réellement disponibles au niveau des côtes bretonnes sont celles qui proviennent du houlographe (Datawell directionnelle) située au niveau des Pierres Noires au sud de l'archipel de Molène (fig. 2 et 12). Celui-ci mesure la mer totale (mer du vent

3. Cartes des niveaux extrêmes (Bretagne nord, Bretagne ouest et Bretagne sud) fournies par le SHOM (2007). 

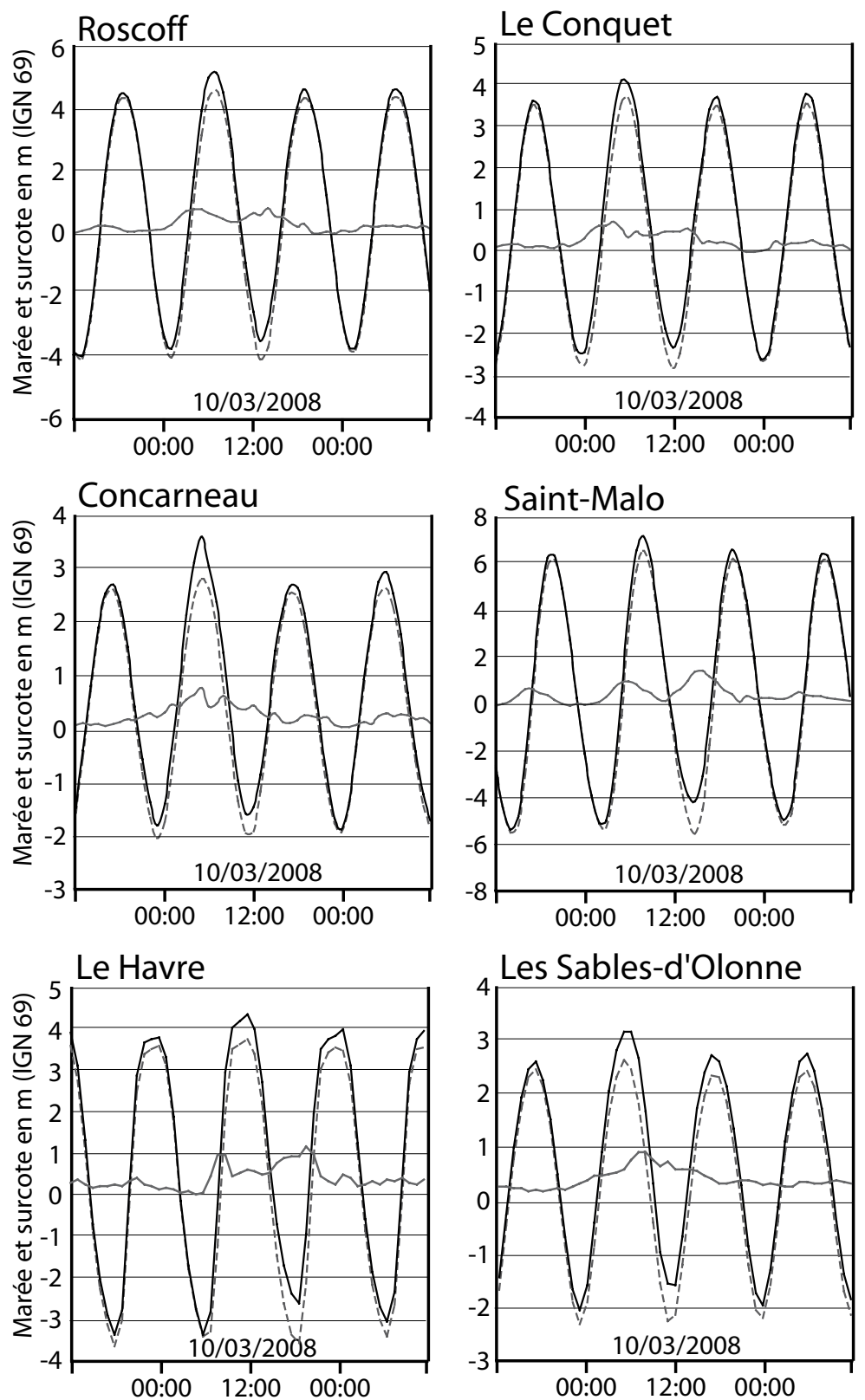

\section{---- Marée prédite — Marée observée — Surcote}

Figure 11 : Conditions marégraphiques enregistrées aux marégraphes de Roscoff, du Conquet, de Concarneau, de Saint-Malo du Havre et des Sables-d'Olonne (source : SHOM)

Tidal conditions recorded at the tidal gauges of Roscoff, Le Conquet, Concarneau, Saint-Malo and les Sables-d'Olonne (source : SHOM) 
Jean-Marie Cariolet et AL.

générée localement et houles combinées). Il est certain que ces données ne reflètent ni la situation pour l'ensemble des côtes bretonnes où les conditions d'agitation ont pu être différentes, ni les conditions au large, mais elles permettent de mieux cerner la puissance de la houle qui a atteint les côtes lors de cette journée du 10 mars 2008. Les variations spatiales sont par ailleurs accessibles par les résultats de la modélisation numérique (fig. 13).

Le 9 mars 2008, veille de la tempête, une houle de provenance ouest-nord-ouest avec une hauteur significative $\left(\mathrm{H}_{\mathrm{mo}}\right)$ de 3 à $4 \mathrm{~m}$ et une période $\left(\mathrm{T}_{\text {pic }}\right)$ d'environ 14 secondes est enregistrée au sud de l'archipel de Molène (fig. 12). Dans la nuit du 9 au 10 mars, à 23 heures UTC, la mer totale change brusquement de direction et passe au secteur sud-ouest et la période chute brusquement à 7 secondes. Ceci est dû à une augmentation significative de la vitesse du vent. Le facteur «mer du vent » est maintenant supérieur par rapport au facteur houle. De 3 heures à 12 heures le 10 mars, la mer totale revient progressivement au secteur ouest tandis que la hauteur et la période augmentent. La houle d'ouest s'impose progressivement par rapport à la mer du vent générée localement. Dès lors, la houle grossit pour atteindre une hauteur significative de 11,5 m à $15 \mathrm{~h} 30$ UTC pour une période variant de 14 à 18 secondes et une période de pic atteignant les 19 secondes. Cette très grande période signifie un plus grand flux d'énergie vers la côte par rapport à des périodes usuelles de 10 à 15 secondes, mais aussi une plus grande amplitude des ondes longues associées aux groupes de vagues, amplitude qui est généralement proportionnelle à $\mathrm{H}_{\mathrm{m} 0}{ }^{2} \mathrm{x}$ $\mathrm{T}_{\text {pic }}$. Le flux d'énergie est par ailleurs, un bon indicateur du transport sédimentaire potentiellement engendré à la côte. Enfin, les vagues de grande période induisent des vitesses orbitales sur le fond de la mer qui augmentent fortement avec la période, avec des effets importants sur le remaniement des corps sédimentaires sur l'ensemble du plateau continental et à la côte.

On peut noter que si le maximum de houle est donc passé juste avant la pleine mer du soir, la hauteur significative des houles est restée supérieure à 8 m jusqu'à 21 heures UTC. C'est essentiellement pendant la pleine mer du soir que la houle a joué un rôle très important.

Afin d'analyser la situation pour l'ensemble du littoral breton et de la Manche, d'autres données de houles ont été obtenues par simulation avec le code de calcul WAVEWATCH III ${ }^{\text {TM }}$ (Tolman, 2009) modifié pour utiliser un paramétrage adapté à la génération et dissipation des fortes houles (Ardhuin et al., 2009a, sous presse) (fig. 13 - planche III). Cette simulation a été validée par des observations sur les bouées et bateaux-phare situés plus au large. La validation nous montre que la simulation est proche des pics de tempête avec une légère surestimation en Manche le reste du temps.

Au final, si les valeurs d'agitation enregistrées pendant le passage de la tempête Johanna sont importantes, elles ne revêtent pas non plus un caractère exceptionnel en terme de hauteur. Selon le CETMEF la période de retour des hauteurs maximales de houles enregistrée est de 2 ans (Goasguen, 2008). Quelques mois plus tôt, la tempête du 9 décembre 2007 était accompagnée de houles d'une hauteur significative de $11,7 \mathrm{~m}$ pour une période associé de 16 secondes $^{4}$.

\section{Bref inventaire des impacts morphogéniques}

Le passage de la tempête « Johanna » le 10 mars 2008 a touché de nombreuses communes littorales dans le quart nord-ouest de l'hexagone (fig. 14). Elle n'a pas fait de victime humaine, mais beaucoup de dégâts sont à signaler : des ouvrages endommagés, un trait de côte qui a fortement reculé par endroit, de nombreuses zones basses qui ont été inondées par la mer, des infrastructures portuaires détériorées. Cent quinze communes de la Vendée jusqu'au département de la SeineMaritime ont ainsi obtenu la reconnaissance de l'état de catastrophe naturelle (avec la mention « inondations et chocs mécaniques liés à l'action des vagues ») ${ }^{5}$. La Bretagne (et plus particulièrement le Finistère et le Morbihan) est la région la plus touchée avec 98 communes concernées.

4. Source : SHOM, Houlographe des Pierres Noires.

5. Source : base Gaspar (Gestion Assistée des Procédures Administratives relatives aux Risques naturels et technologiques), [www.prim.net]. 

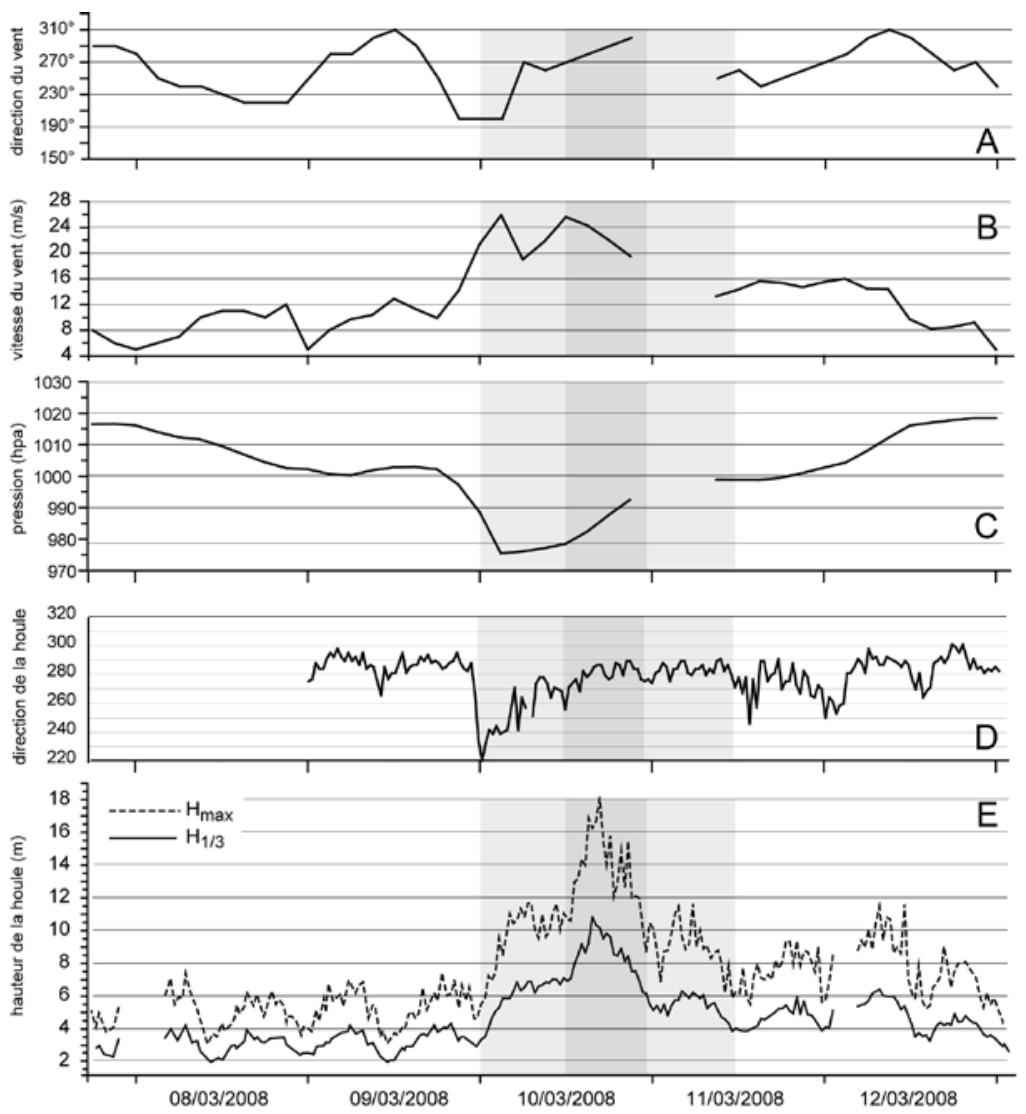

Figure 12 : Conditions météo-marines enregistrées entre le 8 et le 13 mars 2008 au niveau de l'archipel de Molène Les données météorologiques proviennent de la station météorologique du Phare du Stiff (Ouessant) et les données de houle de la bouée datawell des Pierres Noires (sources : Météo-France et SHOM).

Atmospheric and marine conditions recorded between the 8th and the $13^{\text {th }}$ of march 2008 around Molène archipelago

Atmospheric data come from the Stiff Lighthouse weather station (Ushant) and wave data from the Datawell buoy of les Pierres Noires (source : Météo-France and SHOM).

Cette dernière partie propose de dresser un bref inventaire des impacts morphogéniques générés par la tempête. Quelques sites mentionnés ici feront l'objet d'études de cas qui seront traitées dans les articles qui suivent. Ces études de cas illustreront plus précisément les processus morphogéniques qui ont agi lors de cet épisode tempétueux.

Tous les sites inondés durant la pleine mer le matin du 10 mars se situaient sur la côte méridionale de la Bretagne et présentent une orientation sud-sud-est (fig. 14). Les inondations ont eu lieu après le passage du front froid qui a généré de forts vents « d’afflux » de sud-sud-ouest. La pression atmosphérique était alors au plus bas, ce qui explique les valeurs importantes de surcote qui ont été atteintes pendant la pleine mer. À Gâvres (Morbihan), la mer a ainsi franchi la digue inondant une soixantaine d'habitations. Au même moment, les vagues déferlaient littéralement dans les salons de plusieurs maisons de Larmor-Plage (Morbihan) (fig. 14).

Le front froid qui est passé tôt le matin sur la Bretagne, est arrivé rapidement sur le département de la Manche : vers $08 \mathrm{~h} 30$ la rivière le Boscq, gonflée par la marée, est sortie de son lit en plein centre ville de Granville. La mer a franchi une dune à Montmartin-sur-Mer et a envahi le marais. 


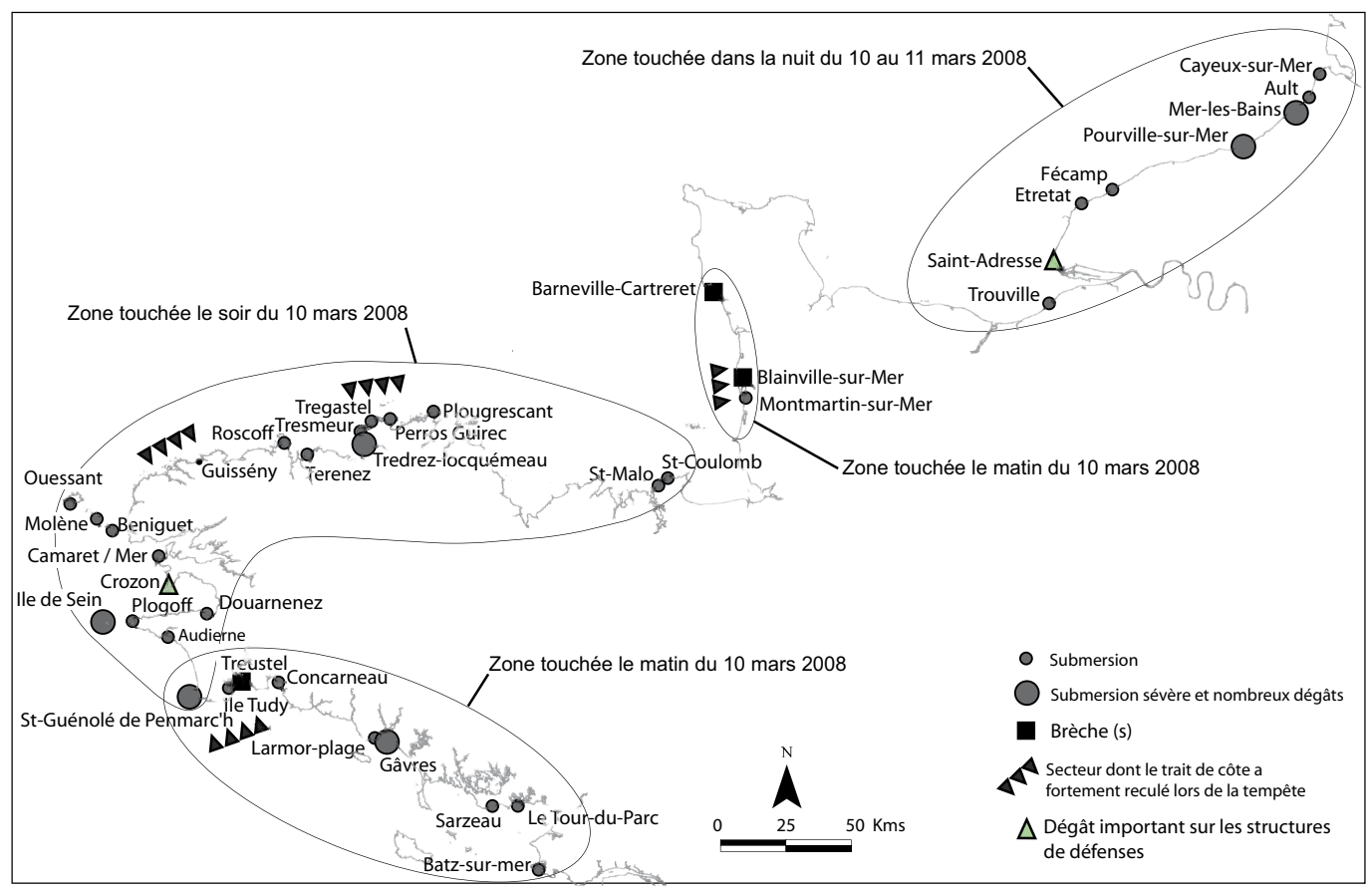

Figure 14 : Carte (non exhaustive) des principaux dégâts recensés après le passage de la tempête "Johanna » (source : Déclarations de dégâts transmises aux DDE, quotidiens Ouest-France, Le Télégramme et Le Trégor)

Map (noexhaustive) of the main damages listed after the passing of the storm "Johanna" (source : damages statement, local newspapers)

Des brèches se sont ouvertes à Carteret, Barneville et Blainville-sur-Mer (fig. 14). À Cherbourg, la Divette, rivière qui se jette dans le port, a débordé, provoquant l'inondation de 35 maisons; les quais du bassin du commerce ont été submergés. La perturbation atmosphérique a pénétré ensuite en baie de Seine puis a progressé vers la côte d'Albâtre et le Pas de Calais, sans provoquer de dégâts, car les vents qui l'accompagnaient n'étaient pas orientés vers la terre, et la surcote associée était relativement déphasée de l'onde de marée (fig. 11).

Durant la pleine mer du soir, la houle et le vent provenaient du secteur ouest. Les dégâts les plus importants se sont produits sur la côte ouest et nord de la Bretagne, au niveau des portions de côte orientés face aux vents d'afflux et à la houle. C'est le cas notamment de la région du Trégor (Côtesd'Armor), orientée à l'ouest, qui a été fortement touchée. À Tredrez-Locquémeau, un cordon de galets a ainsi été brutalement arasé par la mer qui a inondé une dizaine d'habitations et coupé une partie du port du continent. Au Sillon de Talbert des phénomènes de recul ont été observés. La pointe finistérienne a été également fortement touchée par la pleine mer du soir (inondation à l'île de Sein, au port de Penmarc'h, à Audierne, île d'Ouessant, etc.). Les portions de la côte du Léon (Nord Finistère) orientées vers le nord-ouest ont également fortement reculé (fig. 14).

Dans la nuit du 10 au 11 mars, c'est surtout la Haute-Normandie et la Picardie qui éprouvent des submersions, à des degrés variés, durant la pleine mer et pendant le passage de la " traîne » active. Une partie des ouvrages de défense de Sainte-Adresse, près du Havre, a été détruite; Étretat a connu quelques dommages en front de mer; à Fécamp, le boulevard qui longe la plage a été envahi par les galets, et de l'eau a pénétré dans des rues annexes. À Pourville-sur-Mer, à l'ouest immédiat de Dieppe, la submersion a été sévère.

À Mers-les-Bains, malgré d’importants travaux de défense réalisés ces dernières années, la mer a inondé un quartier. Vers la baie de Somme, les vagues ont détruit des ouvrages à Ault; elles ont 
inondé notamment un quartier de la ville de Cayeux-sur-Mer, de même que la " route blanche » qui mène à la pointe du Hourdel (fig. 14).

Au final, le nombre de zones basses qui ont été inondés par la mer (36 cas d’inondations recensés) et l'étendue géographique de la zone touchée (des Pays de la Loire à la Picardie) est exceptionnel pour un seul épisode tempétueux.

\section{Discussion}

Le caractère exceptionnel de l'événement tempétueux du 10 mars 2008 résulte essentiellement de la combinaison de trois facteurs indépendants : une surcote importante, une houle puissante et une pleine mer de vive-eau. Il est cependant très difficile d'évaluer la probabilité d'occurrence d'une telle conjonction car ce type de situation est rare. Sur les côtes Bretonnes, le dernier épisode comparable concerne les tempêtes de l'hiver 1989-1990 (Fichaut et Hallégouët, 1989; Hallégouët et Bodéré, 1993). Sur les côtes Normandes, les tempêtes historiques du 11, 26, 27 et 28 février 1990 avaient engendrées plusieurs submersions désastreuses. En plus des grandes marées, des surcotes et de la forte agitation marine, c'était également l'état déficient du stock sédimentaire qui était ici en cause (Caspar, 1990; Costa, 1997; Caspar et al., 2007).

S’il est possible de calculer séparément les périodes de retour de la houle, du vent et des niveaux de marée observée qui ont été atteints lors de la journée du 10 mars (deux ans environ pour le vent et la houle selon G. Léry (2008), de deux à 100 ans pour la marée observée ${ }^{6}$ ), il est en revanche impossible pour le moment d'estimer la période de retour de la conjonction des trois éléments houle-marée-surcote. Il serait intéressant dans le futur de mettre en place une méthode permettant d'estimer la période de retour d'une telle combinaison de phénomènes sur les côtes du Nord-Ouest de la France. Par ailleurs, il est possible que les périodes très longues jouent un rôle important comme c'est le cas pour d'autres phénomènes comme les seiches. Il serait à ce titre intéressant de s'intéresser aux périodes de retour de paramètres composites tels que $\mathrm{H}_{\mathrm{m} 0}{ }^{2} \mathrm{x}$ $\mathrm{T}_{\text {pic }}$ qui donne une indication de l'amplitude des ondes longues et du flux d'énergie associée à la houle, plutôt qu'au seul paramètre $\mathrm{H}_{\mathrm{m} 0}$.

Les phénomènes de marée et de surcote sont encore considérés en France comme des phénomènes distincts (Simon, 1996). L'interaction entre la marée et la surcote a été étudiée dans d'autres pays comme au Royaume-Uni par J.R. Rossiter (1961), D. Prandle et J. Wolf (1978), Wolf (2009) au Canada et au États-Unis par N. B. Bernier et K. R. Thompson (2007) ou encore en mer du Nord par K. J. Horsburgh et C. Wilson (2007). Ces études ont montré qu'il pouvait exister une interaction significative entre la marée et la surcote à certains endroits du littoral. En France, une telle étude statistique consisterait à comparer les probabilités de distributions des surcotes à différents stades de la marée pour différents points du littoral, puis à observer si les valeurs extrêmes de surcote ont tendance à apparaître lors d'une phase particulière de la marée.

Concernant le rôle joué par les fronts froids lors de l'épisode du 10 et 11 mars 2008, on remarque premièrement que les niveaux d'eau les plus importants ont été atteints juste après le passage du front froid pendant la pleine mer du matin le 10 mars 2008. En outre, toutes les submersions ayant eu lieu le matin du 10 mars ont débuté quelques minutes après le passage du front froid. Deuxièmement, les submersions subies en Bretagne le soir du 10 mars et en Manche la nuit du 10 au 11 mars, ont été précédées par le passage d'un front froid secondaire. Avant de pouvoir affirmer que le front froid est un élément essentiel, il conviendrait de voir dans quelle proportion celui-ci influence les submersions sur nos côtes. Pour cela, il faudrait observer si ce facteur est récurrent lors d'autres épisodes de submersion. R. Caspar et al. (2007) ont déjà mené ce genre d'étude pour le littoral situé entre l'estuaire de la Seine et la baie de Somme. Selon les auteurs il apparaît que près de $70 \%$ des submersions se produisent peu après le passage d'un front froid actif. Ce dernier

6. Goasguen (2008) et cartes des niveaux d'eau extrêmes fournies par le SHOM. 
Jean-Marie Cariolet et AL.

engendre de forts vents d'afflux d'ouest à nord-ouest qui creusent rapidement la mer, inondant les zones basses.

Afin d'expliquer les impacts morphogéniques causés par une tempête, il faut non seulement prendre en considération les conditions météo-marines mais également l'état des stocks sédimentaires au moment de l'événement. Ce paramètre morphologique détermine la capacité des milieux littoraux à encaisser l'impact d'une tempête (Orford, 1977; Paskoff, 1985 ; Carter, 1988; Carter et Orford, 1993; Costa, 1997; Sabatier et al., 2009). La tempête « Johanna » a touché le littoral de l'ouest de la France en mars, à la sortie de l'hiver. L'état des stocks sédimentaires sur certaines portions du littoral était peut-être déficitaire à cette période de l'année, ce qui pourrait expliquer les nombreux impacts morphogéniques recensés. Cependant, la fragilisation d'une portion littorale semble pouvoir s'opérer dans un temps beaucoup plus court. F. Sabatier et al. (2009) indiquent ainsi que sur la plage de Vert-Bois (île d'Oléron), le 10 mars 2008, la dune a d'abord été amincie par l'action du vent ce qui aurait permis aux vagues d'atteindre la dune alors fragilisée pendant la marée haute, quelques heures plus tard. Enfin, certaines portions du littoral peuvent présenter une certaine fragilité face aux événements météo-marins exceptionnels. On remarque par exemple que tous les sites qui ont été inondés le matin du 10 mars en Bretagne présentaient une orientation sud-est (Gâvres, Île Tudy, Larmor-Plage, Sarzeau, Le Tour-du-Parc) (fig. 15). On peut supposer que ces secteurs d'habitude en position d'abri (les houles principales au sud de la Bretagne provenant du secteur ouest-sud-ouest) présentent une certaine fragilité face à des conditions météo-marines peu clémentes. Le profil de plage des secteurs touchés n'était peut-être pas adapté à de telles conditions, ce qui pourrait expliquer le nombre important de submersions sur les sites orientés vers le sud-est.

De nombreux auteurs ont observé sur les côtes du Nord-Ouest de la France une variabilité des événements morphogènes qui semble s'organiser selon des cycles de 10 à 12 ans. À partir de l'analyse des données de houles enregistrées au large de Ouessant, B. Fichaut et S. Suanez (2008) constatent ainsi que la décennie 1979-1990 est marquée par une fréquence élevée d'épisodes morphogènes, alors que la période 1990-2002 est marquée par une diminution de ce type d'épisodes. S. Costa (1997) parle de la période 1975-1990 comme d'une période propice aux submersions sur le littoral Haut Normand, et souligne également l'inversion de la tendance en 1990. B. Hallégouët et A. Hénaff (2006) ont eux observé sur la côte sud du Finistère une période de calme et de régénération des systèmes littoraux entre 2000 à 2006, phase également constatée par P. Stéphan (2008) sur le Sillon de Talbert (Côtes-d'Armor) et en Rade de Brest. Enfin, les années 2007 et 2008 semblent marquer le début d'une nouvelle période morphogène. Avec les tempêtes du 20 mars 2007 ou encore du 10 février 2009, la tempête « Johanna » s'inscrit pleinement dans cette phase de renforcement des conditions cycloniques.

P. A. Pirazzoli et al. (2004) indiquent que le caractère cyclique des phases morphogènes en Bretagne serait lié à une fluctuation de l'indice d'oscillation nord atlantique (ou NAO, pour North Atlantic Oscillation). Les phases morphogènes se produiraient lorsque l'indice est négatif et les phases de calme lorsque celui-ci est positif. Quelques auteurs ont néanmoins démontré que cet indice ne pouvait expliquer à lui seul ces phases cycliques (Betts et al., 2004).

\section{Conclusion}

Cet article s'est proposé d'apporter un éclaircissement sur les conditions météo-marines qui ont engendré de nombreux dégâts sur le littoral du Nord-Ouest de la France le 10 et 11 mars 2008. Les développements récents dans le domaine de la météorologie dynamique, portant notamment sur le courant-jet et l'interaction barocline, permettent de mieux comprendre la genèse et le renforcement des tempêtes en Atlantique. L'intégration de ces données permet ainsi une analyse plus poussée de la tempête du 10 et 11 mars 2008. En outre, le lien entre les fronts froids et les submersions marines semble se préciser, du moins en ce qui concerne certaines portions du littoral du Nord-Ouest de la France. 
Les communes littorales concentrent de plus en plus d'enjeux sur leur territoire ce qui augmente d'autant plus les risques engendrés par les tempêtes. La gestion de ces espaces devient donc quelques fois difficile, notamment dans le contexte actuel d'élévation du niveau marin et de dérèglement climatique, qui prévoit une augmentation de la fréquence des événements tempétueux. Au final, la tempête du 10 et 11 mars 2008 peut être considérée comme un événement de référence, qui aidera sûrement les services de l'État (DDE) à réévaluer les zonages réglementaires des Plans de Prévention des Risques par Submersion Marine, ou encore à redimensionner certains ouvrages de défense. Une analyse complète des aspects météo-marins de cette tempête semblait donc nécessaire. Pour finir, comme il a été évoqué précédemment, les articles qui vont suivre traiteront plus précisément de quelques cas d'étude d'impacts morphogéniques cités dans ce texte introductif.

\section{Remerciements}

Merci à Marc Robin (Géolittomer-UMR 6554 CNRS LETG) pour les données météo-marines relatives aux régions Pays de la Loire et Poitou-Charentes. Merci à Philippe Arbogast de Météo France pour ses remarques. Nous remercions également Guy Wöppelmann, responsable du projet SONEL, pour la mise à disposition des données marégraphiques observées. Merci enfin à Serge Suanez pour ses conseils et ses commentaires constructifs.

\section{Références}

Ardhuin F., Chapron B., Collard F., 2009a. Observation of swell dissipation across oceans, Geophysical Research Letters, vol. 36, p. L06607.

Ardhuin F., Marié L., Rascle N., Forget P., Roland A., Observation and estimation of Langrangian Stockes and Eulerian currents induced by wind and waves at the sea surface, Journal of Physical Oceanography (sous presse) [http://hal.archives-ouvertes.fr/hal-00331675/].

Ayrault F. et Joly A., 2000. Une nouvelle typologie des dépressions atmosphériques : classification des phases de maturation, Comptes Rendus de l'Académie des Sciences, Sciences de la terre et des planètes, vol. 330, p. 167-172.

Baehr C., Poupoonneau B., Ayrault F., Joly A., 1999. Dynamical characterization of the FASTEX cyclogenesis cases, the Quaterly Journal of the Royal Meteoroogical Society, vol. 125, n 561, p. 3469-3494.

Beltrando G., 2004. Les climats : processus, variabilité et risques, Armand Colin, 261 p.

Bernier N. B., Thompson K. R., 2007. Tide-surge interaction off the east coast of Canada and northeastern United States, Journal of Geophysical Research, vol. 112, p. 1-12.

Bessemoulin P., 2002. Les tempêtes en France, Annales des Mines, p. 9-14.

Betts N. L., Orford J. D., White D., Graham C. J., 2004. Storminess and surges in the south-western approaches of the eastern North Atlantic : the synoptic climatology of recent extreme coastal storms, Marine Geology, vol. 210, n 1-4, p. 227-246.

Bouligand R., Tabeaud M., 1998. Cent ans de surcotes à Brest, Met Mar, n 180, p. 12-16.

Carter R. W. G., 1988. Coastal environments : an introduction to the physical, ecological and cultural systems of coastlines, Academic Press, London, 617 p.

Carter R. W. G., Orford J. D., 1993. The morphodynamics of coarse clastic beaches and barriers : a shortand long-term perspective, Journal of Coastal Research, n 15, SI, p. 158-179.

Caspar R., 1988. Marée de tempête sur le littoral de Haute-Normandie, 22 janvier 1988, Met Mar, n 141, p. $12-18$.

—, 1990. Marée de tempête dans le nord-ouest de la France, Neptunia, Amis des musées de la Marine, Paris, $\mathrm{n}^{\circ} 178$, p. 8-15.

—, 2009. Les submersions de tempête de la nuit du 10 au 11 mars 2008 entre l'estuaire de la Seine et la baie de Somme, Météo-France, Document interne, (non publié). 
Jean-Marie Cariolet et al.

Caspar R., Costa S., Jаков E., 2007. Fronts froids et submersions de tempête dans le nord-ouest de la France; le cas des inondations par la mer entre l'estuaire de la Seine et la baie de Somme, La Météorologie, $n^{\circ} 57$, p. 37-47.

Costa S., 1995. Vulnérabilité des villes côtières de Haute-Normandie et de Picardie face à l'élévation du niveau marin, Hommes et terres du Nord. "Les milieux littoraux », vol. 1-2, p. 48-57.

—, 1997. Dynamique littorale et risque naturel : l'impact des aménagements, des variations du niveau marin et des modifications climatiques entre la baie de Seine et la baie de Somme. Thèse de doctorat, Université de Paris-1, 376 p.

Costa S., Cantat O., Pirazzoli P. A., Lemaître M., Delahaye D., 2004. Vents forts et submersions de tempête en Manche Orientale : analyse météo-marine sur la période historique récente, Actes du Colloque de l'Association Internationale de Climatologie "Climat, mémoire du temps. Les relations climat-espacesociété », p. 277-280.

Daniel P., 2001. Cartographie des zones à risque de surcotes sur les côtes de la Manche et de l'Atlantique. Météo-France, rapport interne, 80 p., (non publié).

Fichaut B., Hallegouet B., 1989. Banneg : une île dans la tempête, Penn ar Bed, nº 135, p. 36-43.

Fichaut B., Suanez S., 2008. Les blocs cyclopéens de l'île de Banneg (archipel de Molène, Finistère) : accumulations supratidales de forte énergie, Géomorphologie : relief, processus, environnement, $\mathrm{n}^{\circ}$ 1, p. 15-32.

Goasguen G., 2008. Finistère - Demande de reconnaissance de l'état de catastrophe naturelle - Action des vagues - Tempête du 10 mars 2008, Rapport technique pour la préfecture du Finistère, Centre d'Etudes Techniques Maritimes et Fluviales, Département Environnement Littoral et Cours d'Eau, 25 mars 2008, $6 \mathrm{p}$, (non publié).

Hallegouet B., Bodere J.-C., 1993. Un littoral fragilisé : le sud de la Baie d'Audierne, Le Pays Bigouden à la croisée des chemins, Cap Caval, suppl. 17, p. 263-271.

Hallegouet B., Henaff A., 2006. Évolution récente et gestion des espaces littoraux de l'ouest Cornouaille. "Quelles pistes de développement pour le territoire? » Actes des rencontres de l'ouest Cornouaille, mai-juin 2005, Association Ouest Cornouaille Promotion, Pont-L'abbé, p. 20-34.

Hequette A. et Vasseur B. 1998. Storm surges and erosion of coastal dunes between 1957 and 1988 near Dunkerque (France), Southwestern North Sea, dans Pye K., Allen J. R. L. (dir.), Coastal and Estuarine Environments, Geological Society, London, Special Publications, n 175, p. 99-107.

Hontarrede M. et Galli F.-L., 2001. Des inondations meurtrières, exemples de marées de tempêtes à travers le monde, Met Mar, n 190, p. 18-21.

Horsburgh K. J., Wilson C., 2007, Tide-surge interaction and its role in the distribution of surge residuals in the North Sea, Journal of Geophysical Research, vol. 112, p. 1-13.

Joly A., 1995. Le front polaire : un concept dépassé qui a la vie dure, La Recherche, nº 273, p. 128-135.

Leny G., 2008. Rapport météorologique, procédure de reconnaissance de catastrophe naturelle, " phénomènes liés à l'action de la mer », période : tempête du 10 mars 2008, Rapport Météo-France - Centre Départemental du Finistère, 3 avril 2008, 3 p.

Longuet-Higgins M. S., Stewart R. W., 1963. A note on wave set-up, Journal of Marine Research, vol. 21 , $\mathrm{n}^{\circ} 1$, p. 4-10.

Morton R. A., 2002. Factors controlling storm impacts on coastal barriers and beaches - apreliminary basis for real-time forecasting, Journal of Coastal Research, vol. 18, n³ 3, p. 486-501.

Okimiro M., Guza R. T., Seymour R. J., 1993. Excitation of seiche observed in a small harbor, Journal of Geophysical Research, vol. 98, p. 18201-18211.

Orford J. D., 1977. A proposed mechanism for storm beach sedimentation, Earth Surface Processes, vol. 2, $\mathrm{n}^{\circ} 4$, p. 381-400.

Paskoff R., 1985. Les littoraux : impact des aménagements sur leur évolution, Masson, Paris, 184 p.

Pirazzoli P. A., 2000. Surges atmospheric pressure and wind change and flooding probability on the Atlantic coast of France, Oceanologica Acta, vol. 23, n 6, p. 643-661.

Pirazzoli P. A., Regnauld H., Lemasson L., 2004. Change in storminess and surges in western France during the last century, Marine Geology, vol. 210, p. 307-323. 
Pirazzoli P. A., Costa S., Dornbusch U., Tomasin A., 2006. Recent evolution of surge-related events and assessment of coastal flooding risk on the eastern coast of the English Channel, Ocean Dynamics, vol. 56, n 5-6, p. 498-512.

Prandle D., Wolf J., 1978. The interaction of surge and tide in the North Sea and River Thames, Geophys. J. R. Aston. Soc., vol. 55, p. 203-216.

Regrain R., 1992. Une station touristique confrontée au recul de la falaise : Ault, Les Littoraux en France : risques et aménagements. Amiens : Centre Régional de Documentation Pédagogique et Association des Professeurs d'Histoire et de Géographie, 97 p.

Rossiter J. R., 1961, Interaction between tide and surge in the Thames, Geophysical Journal of Royal Astonomical Society, vol. 6, p. 29-53.

Sabatier F., Anthony E. J., Hequette A., Suanez S., Musereau J., Ruz M. H., Regnauld H., 2009. Morphodynamics of beach/dune systems : examples from the coast of France, Géomorphologie : relief, processus, environnement, $\mathrm{n}^{\circ} 1$, p. 3-22.

Santurette P., 2001. A propos des fronts froids, Met Mar, n 191, p. 8-13.

Simon B., 1996. Détermination des hauteurs d'eau extrêmes pour la délimitation du domaine public maritime, Annales Hydrographiques, vol. 20, n 767, p. 17-43.

Stephan P., 2008. Les flèches de galets de Bretagne: morphodynamiques passée, présente et prévisible, thèse de doctorat, Université de Bretagne Occidentale, 560 p.

Suanez S., Cariolet J.-M. L'action des tempêtes sur l'érosion des dunes : les enseignements de la tempête du 10 mars 2008, Norois, ce volume.

ToLman H. L., 2009. User manual and system documentation of WAVEWATCH III ${ }^{\mathrm{TM}}$, version 3.14, Tech Rep. 276, NOAA/NWS/NCEP/MMAB.

Tonnerre M.-A., 2001. Contributions à l'étude des tempêtes dans la Manche et en façade atlantique de la France, au nord de l'île de Noirmoutier, 1965-1994, Doctorat d'État, Lille USTL, 363 p.

Tonnerre-Guerin M.-A., 2003. Les tempêtes, un concept et une genèse revisités : l'exemple de la façade occidentale de l'Europe, Annales de géographie, vol. 112, nº 633, p. 451-470.

Wolf J., 2009. Coastal flooding : impacts of coupled wave-surge-tide models, Natural Hazrads, vol. 49, p. 241-260.

Cet article a été reçu le 3 juillet 2009 et définitivement accepté le 25 janvier 2010. 
planche I (Jean-Marie CARIOLET et al. - Aspects météo-marins de la tempête du 10 mars 2008...)

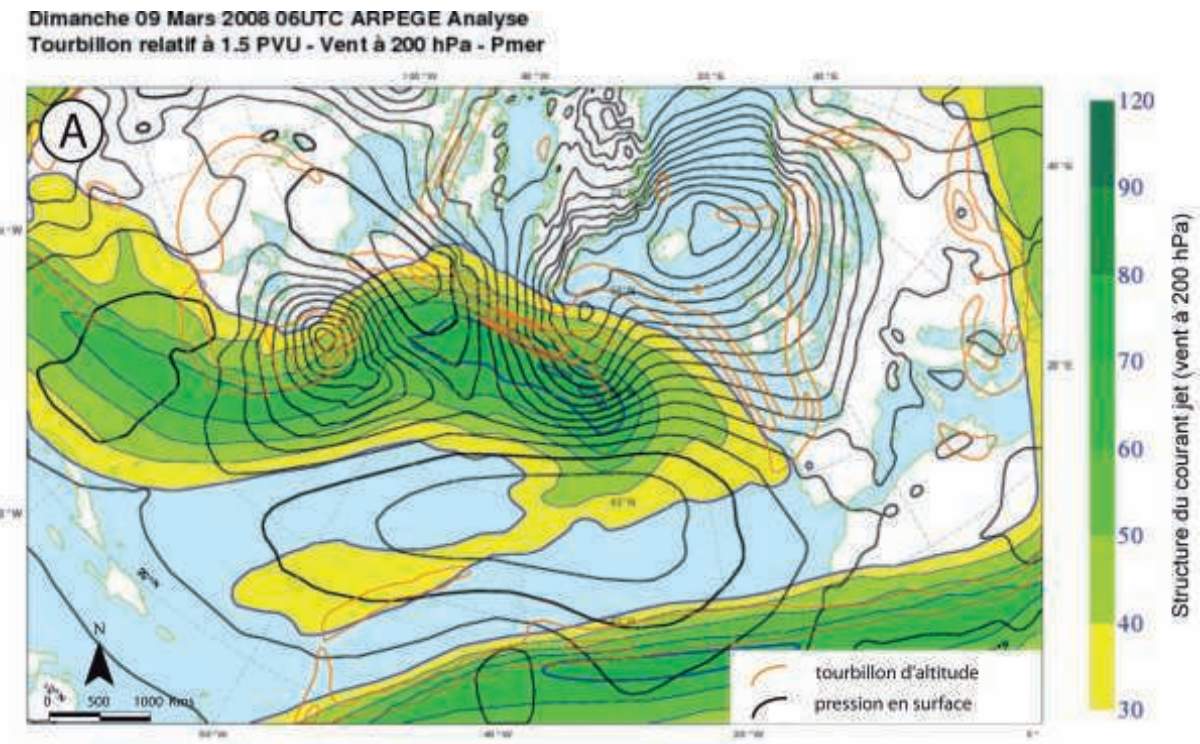

O9MAR2O08 00Z

$850 \mathrm{hPa}$ Temperatur (Grad C)
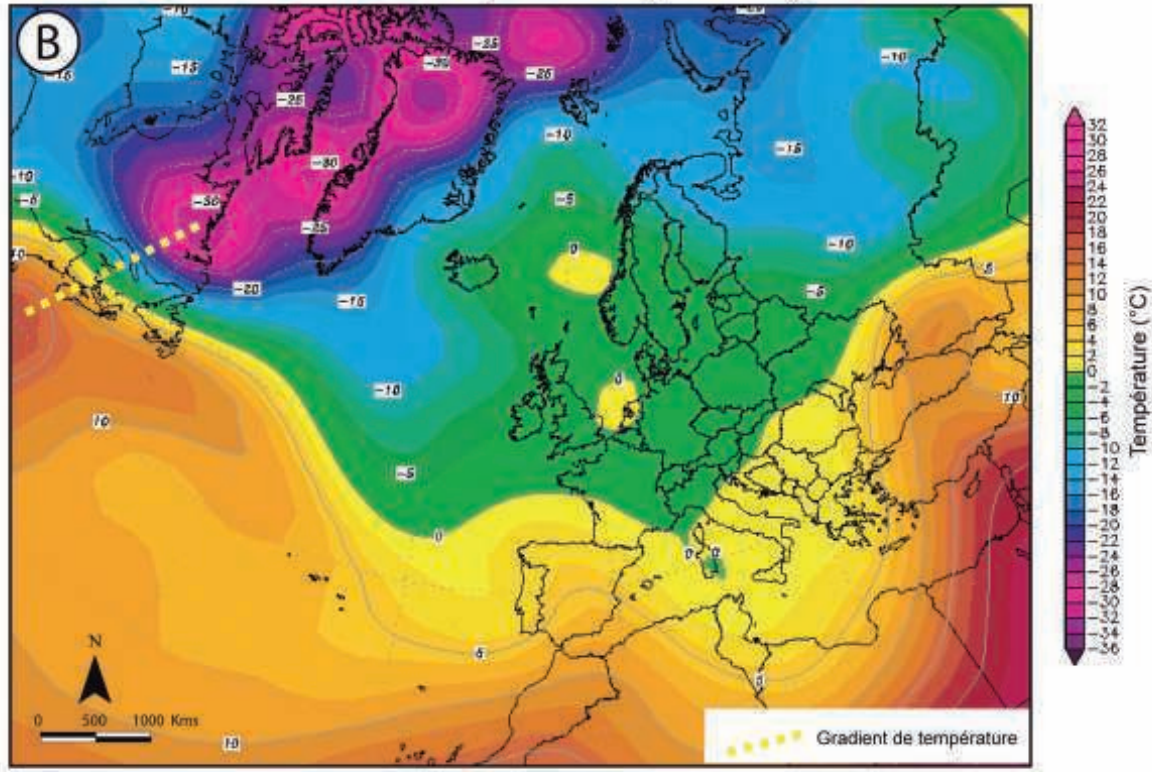

Daten: Reanalysis des NCEP

(C) Wetterzentrale

www wetterzentrale, de

Figure 7 : A : Carte issue du modèle global de Météo-France représentant les vents à $200 \mathrm{hPa}$ (on remarque la structure du courant jet en vert), la pression en surface (isolignes noires) et les tourbillons d'altitude (isolignes orange) le 9 mars 2008 à 06 h 00 UTC. B : carte de la température à 850 hPa le 9 mars 2008 à 00 h 00 UTC. On remarque le très fort gradient de température au niveau de la côte est du Canada qui « alimente » le courant-jet (source : www.wetterzentrale.de/topkarten/fsreaeur.html)

A : Chart showing wind at $200 \mathrm{hPa}$ (we can observe jet stream in green), surface pressure and vortex the $9^{\text {th }}$ of march 2008 at 06 heures UTC. B : chart of temperature at 850 hPa the 9th of march 2008 at 00:00 UTC. We observe the huge temperature gradient around the East coast of Canada, which power the jet stream (Source : www.wetterzentrale.de/topkarten/fsreaeur.html) 
planche II (Jean-Marie CARIOLET et al. - Aspects météo-marins de la tempête du 10 mars 2008...)

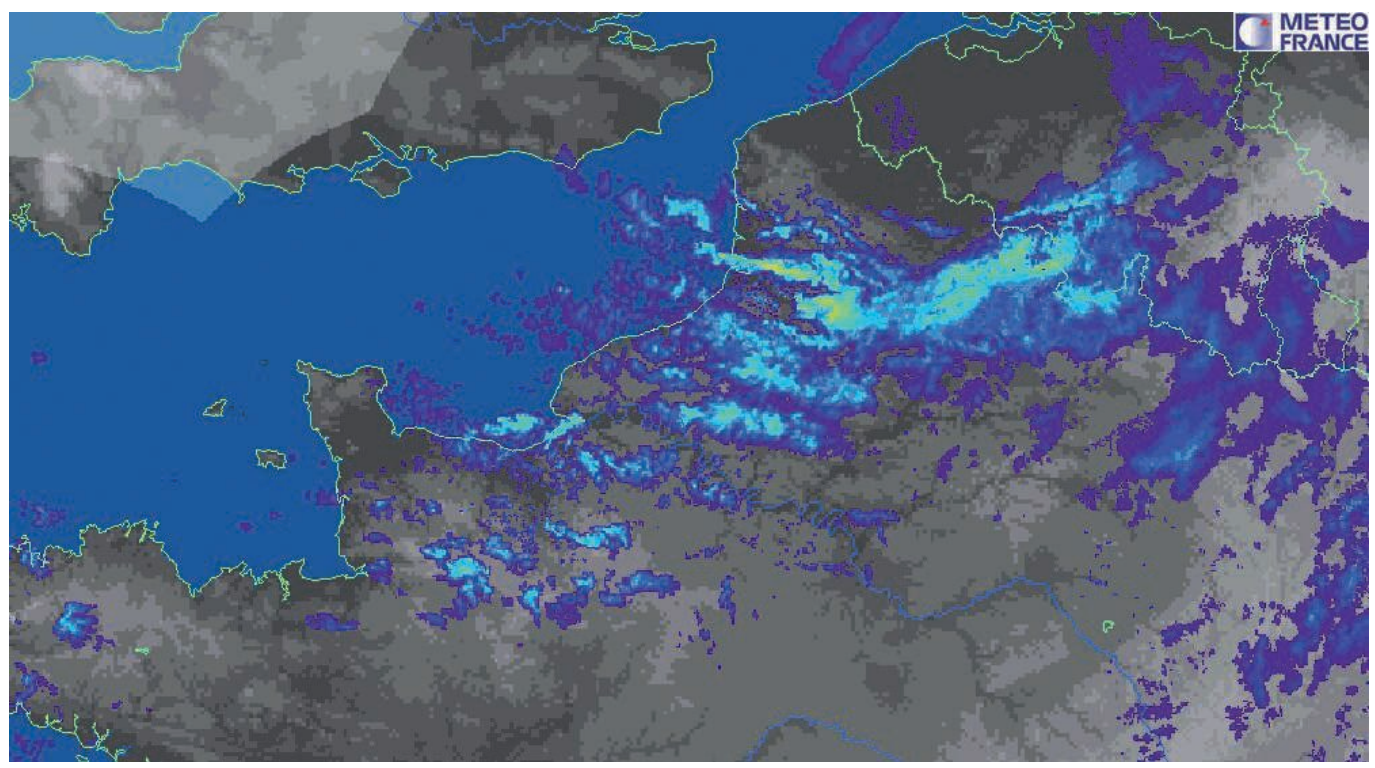

Figure 9 : Image radar d'une partie du Nord-Ouest de la France le 11 mars 2008 à 00 h 30 UTC (au moment des submersions de la côte d'Albâtre) : pas d'entité météorologique définie mais plutôt une « traîne " en Manche qui persiste en raison d'un léger forçage d'altitude (source : Météo-France)

Radar image of a part of North West of France the 11th of march 2008 at $00 \mathrm{~h} 30$ UTC (during inundations on the côte d'Albâtre) : any meteorological entity defined but a residue in the Channel which persist because of a light altitude forcing (source : Météo-France)
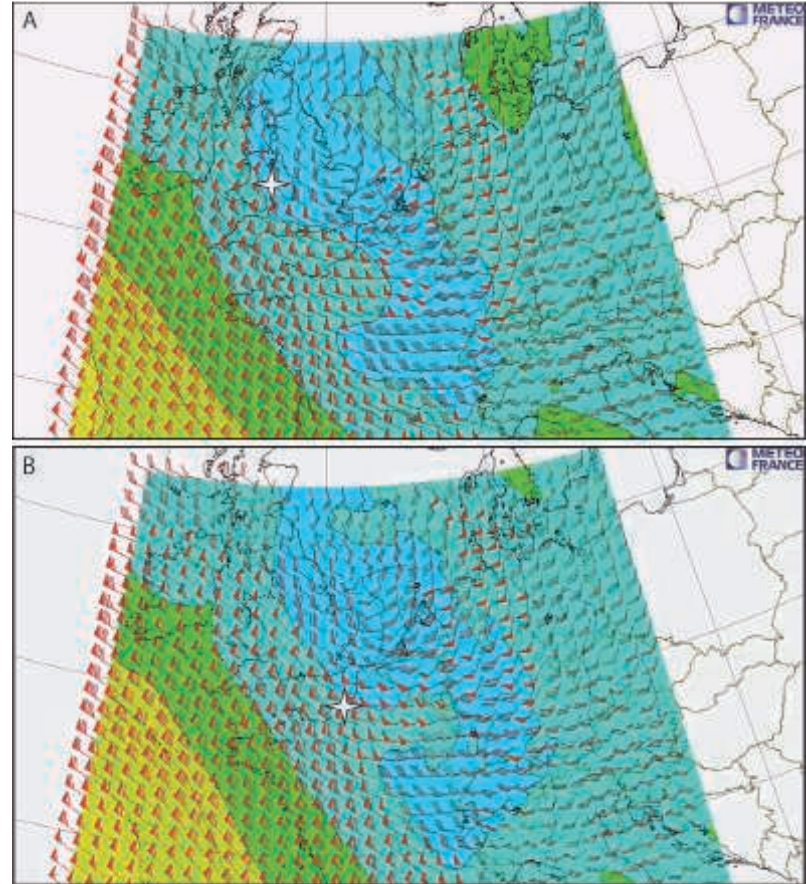

$-32<$ $<-28<$ $<-24<$ $<-20<$ $<-16<$
Figure 10 : Évolution de la température et des vents à $500 \mathrm{hPa}$ prévue par le modèle «Aladin » (base 12 heures UTC le 10 mars 2008), pour le 10 mars à 21 heures UTC (a) et le 11 mars à 00 h UTC (b).

Une «bulle » de l'ordre de -30 degrés (étoile), située sur le Pays de Galles pour 21 heures UTC est visible dans les parages de la baie de Somme pour $00 \mathrm{~h}$ UTC. Son passage en Manche renforcera l'instabilité de la masse d'air et l'intensité des rafales de vent (source : Météo-France)

Temperature and wind at 500 hPa evolution calculated by the model "Aladin" (base 12 heures UTC the $10^{\text {th }}$ of march 2008), for the $10^{\text {th }}$ of march at 21 heures UTC $(a)$ and the 11 march at 00 h UTC (b). A 30 degrees "bubble" (star), located on Wales at 21:00 UTC is visible around the Baie de Somme at 00 h UTC (source : Météo-France). 


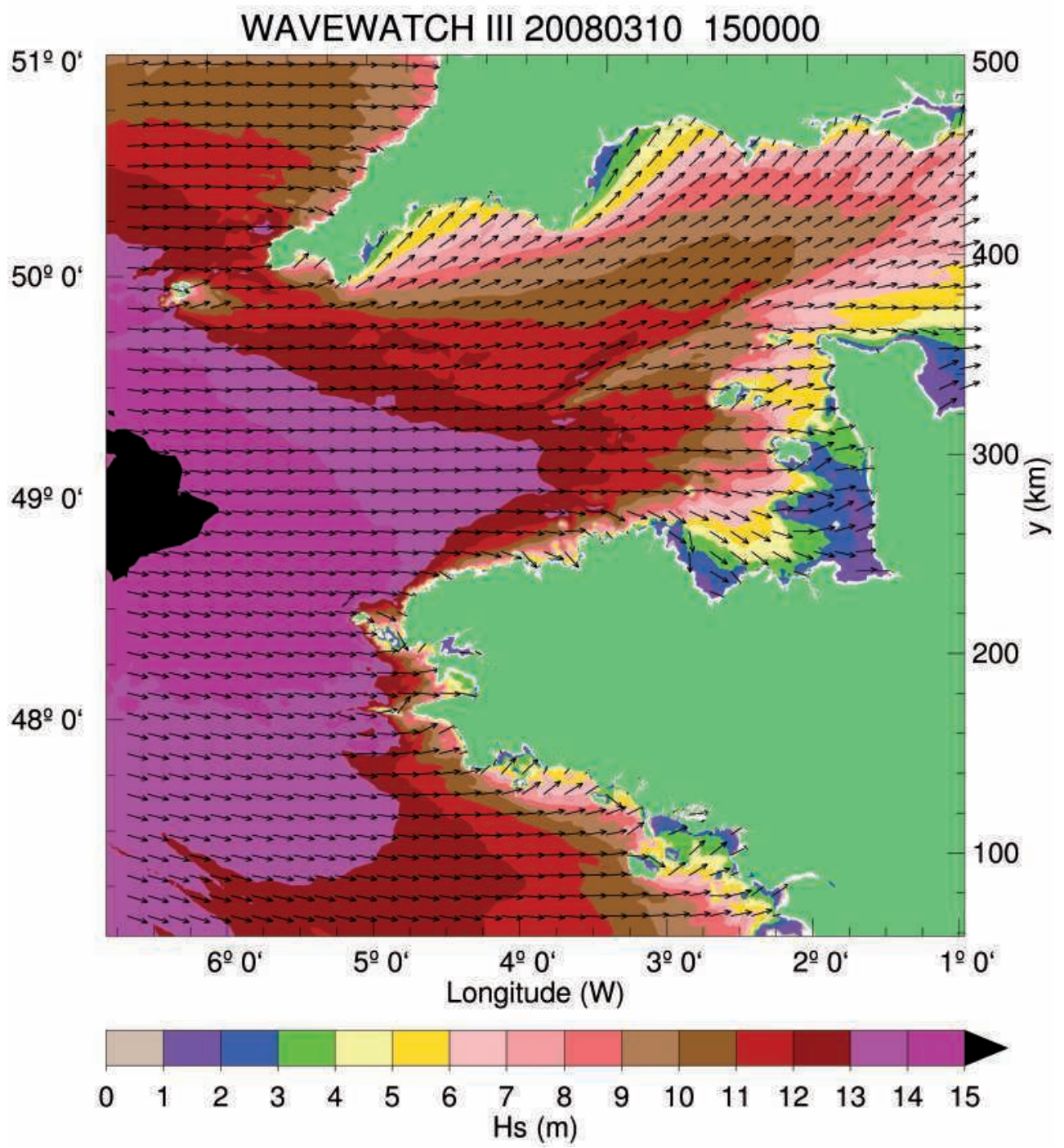

Figure 13 : Simulation des conditions d'agitation au niveau de la Bretagne et de la Manche le 10 mars 2008 à 15 h 00 UTC (la simulation a été obtenue grâce au modèle numérique WAVEWATH III ${ }^{\mathrm{TM}}$ [Source : SHOM])

Wave conditions simulation around Brittany and in the Channel the 10th af march 2008 at 15 h00 UTC (the simulation has been obtain with WAVEWATH III ${ }^{\mathrm{TM}}$ numerical model [Source: SHOM]) 